University of Texas at El Paso

ScholarWorks@UTEP

Open Access Theses \& Dissertations

2020-01-01

\title{
Validity Of A Portable X-Ray Fluorescence Device For Analyzing Field Dust Wipe Samples For Lead
}

Alexander Boakye Obeng

University of Texas at El Paso

Follow this and additional works at: https://scholarworks.utep.edu/open_etd

Part of the Public Health Education and Promotion Commons

\section{Recommended Citation}

Obeng, Alexander Boakye, "Validity Of A Portable X-Ray Fluorescence Device For Analyzing Field Dust Wipe Samples For Lead" (2020). Open Access Theses \& Dissertations. 3114.

https://scholarworks.utep.edu/open_etd/3114

This is brought to you for free and open access by ScholarWorks@UTEP. It has been accepted for inclusion in Open Access Theses \& Dissertations by an authorized administrator of ScholarWorks@UTEP. For more information, please contact Iweber@utep.edu. 


\title{
VALIDITY OF A PORTABLE X-RAY FLUORESCENCE DEVICE FOR ANALYZING FIELD DUST WIPE SAMPLES FOR LEAD
}

\author{
ALEXANDER BOAKYE OBENG \\ Master's Program in Public Health
}

APPROVED:

Christina Sobin, Ph.D., Chair

Gabriel Ibarra-Mejia, MD., Ph.D., MSErg

Ganga Hettiarachchi, Ph.D.

William Hargrove, Ph.D.

Stephen L. Crites, Jr., Ph.D.

Dean of the Graduate School 


\section{DEDICATION}

I dedicate my work to my beloved mother, Evelyn Nimako, who has been my source of inspiration and gave me strength when I thought of giving up.

To Mr. and Mrs. Ampong who taught me that it is never too late to change careers to pursue your true passion. 
FIELD DUST WIPE SAMPLES FOR LEAD

by

ALEXANDER BOAKYE OBENG, B.S.

\author{
THESIS \\ Presented to the Faculty of the Graduate School of \\ The University of Texas at El Paso \\ in Partial Fulfillment \\ of the Requirements \\ for the Degree of
}

MASTER OF PUBLIC HEALTH

Department of Public Health

THE UNIVERSITY OF TEXAS AT EL PASO

August 2020 


\section{ACKNOWLEDGEMENTS}

I would like to give special recognition to Dr. Christina Sobin, and to the Department of Housing and Urban development (HUD) for providing funding for this research. I would like to thank Dr. Gabriel Ibarra-Mejia, Dr. Bill Hargrove, and Dr. Ganga Hettiarachchi for their extraordinary support and advice in this thesis process. Thanks to Michelle Del Rio, Carlos Chavarria, Crystal Costa, Christina Rodriguez, Chandima Wekumbura Gedara and the UTEP Lead Research Team for supporting and participating in the research activities. Again, special thanks to Dr. Carla Campbell and Dr. Christina Sobin, for mentoring and supporting me throughout my MPH program. Dr. Sobin's funding was possible by grants from the National Institute of General Medical Sciences of the National Institutes of Health under linked Award Numbers RL5GM118969, TL4GM118971, and UL1GM118970, Center for Clinical and Translational Science, The Rockefeller University; Paso del Norte Health Foundation, El Paso, Texas; and J. Edward and Helen M. C. Stern Professorship in Neuroscience (CS), University of Texas, El Paso, and by the U.S. Department of Housing and Urban Development, Lead Technical Studies Funding Program, SF424 (CS, PI). The content is solely the responsibility of the authors and does not necessarily represent the official views of the National Institutes of Health and HUD. 


\begin{abstract}
Background and Significance. Interior dust lead loadings on floors and windowsills are reliable predictors of a child's blood lead level and an important predictor in "clearance testing" of residences. The traditional method of determining dust lead levels on surfaces is to send dust wipe samples to a laboratory for analysis. These laboratory analyses are expensive and analysis reports typically take up to 2 weeks to complete. The portable X-ray fluorescence device has been touted as a technique that can provide fast, accurate, and precise results regarding the presence of dust lead hazards in residences but needs to be further evaluated for comparability with laboratory analyses.
\end{abstract}

Methods. Dust wipe samples $(n=109)$ collected from 13 homes were tested by means of X-ray fluorescence (XRF) analysis and subsequently analyzed for lead using Inductively Coupled Plasma Mass Spectrometry (ICPMS). Samples were collected from floors and interior windowsills and included blank samples and samples with known concentrations for quality control.

Results. Considering ICPMS as the "gold standard," the XRF produced an average false negative rate of $5.6 \%$ at the new EPA dust lead hazard standards of $10 \mu \mathrm{g} / \mathrm{ft} 2$ for floors and $100 \mu \mathrm{g} / \mathrm{ft} 2$ for windowsills. Interestingly, there were no false positive results from the XRF device at the new dust lead hazard standards. A Bland Altman analysis showed that $96.3 \%$ of the data points were within the lower and upper acceptable limits of agreement. Results of a MannWhitney $U$ test showed that lead concentration in windowsill dust wipe samples were significantly higher than floor dust wipe samples as reported by the ICPMS device $(\mathrm{U}=475.50 \mu \mathrm{g} / \mathrm{ft} 2, \mathrm{p}<0.001)$. 
Conclusion. The results of the study suggested that the XRF device has a good agreement with the ICPMS device at lower lead concentrations and may be appropriate for measuring lead concentrations in field dust wipe samples from homes where lead concentrations are not high. It can also be concluded that XRF device may be used as a positive and negative screen for lead dust hazards in the homes of children. Additional studies are needed to further evaluate the accuracy and comparability of XRF devices at the new EPA dust lead hazard standards. 


\section{TABLE OF CONTENTS}

Page

ACKNOWLEDGEMENTS .......................................................

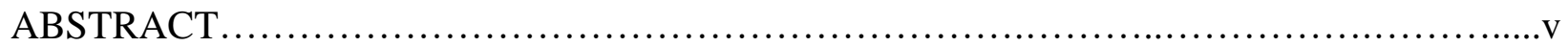

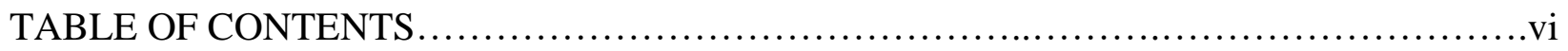

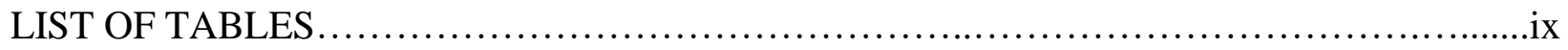

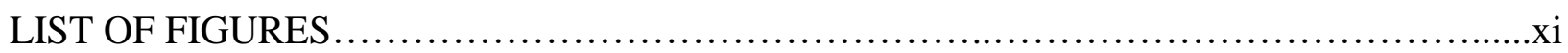

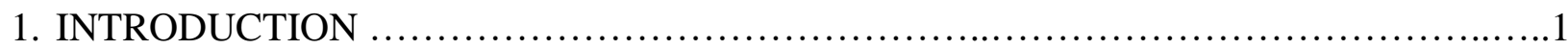

1.1 Effects of Childhood Lead Exposure............................................

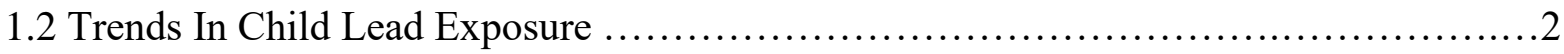

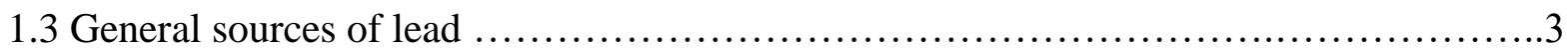

1.4 Lead Exposure In The Home ...............................................6

1.5 Importance Of Lead Dust As An Indicator of Lead Hazards In The Home ...............7

1.6 EPA Dust Lead Hazard Standards..............................................

1.7 Currently Available Methods For Testing For Lead Dust Hazard Sources In The Home

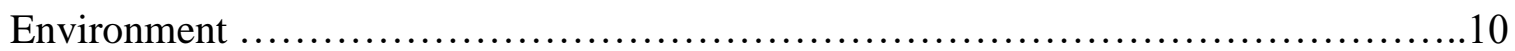

1.7.1 Home Spot Lead Test Kits ..........................................10

1.7.2 Laboratory Tests ....................................................... 14

1.7.2.1 Atomic Absorption Spectrometry (AAS) .................................

1.7.2.2 Graphite Furnace Atomic Absorption Spectroscopy (GFAAS) ..........17

1.7.2.3 Inductively Coupled Plasma Mass Spectrometry (ICP-MS) ...........22

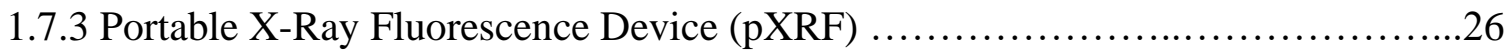

1.7.4 Comparison of pXRF And Laboratory Techniques for Analyzing Environmental

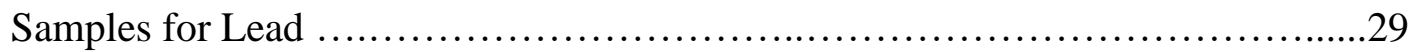




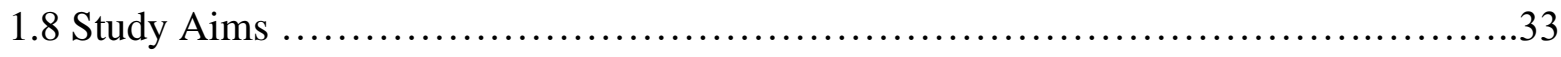

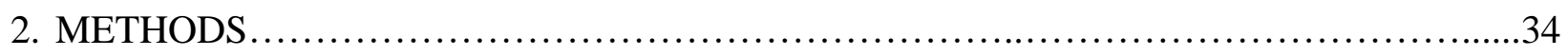

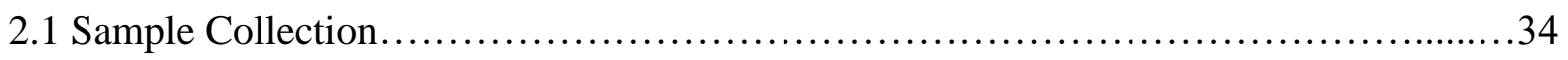

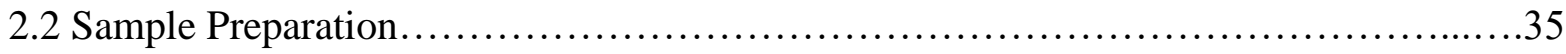

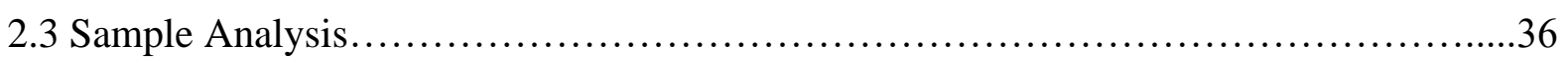

2.3.1 Portable XRF Analysis of Samples ............................................

2.3.2 ICP-MS Analyses of Samples................................................

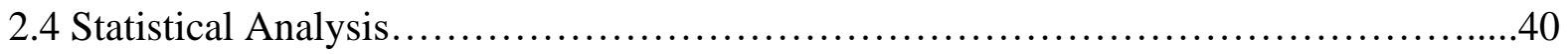

2.4.1 Bland Altman Analysis.......................................................40

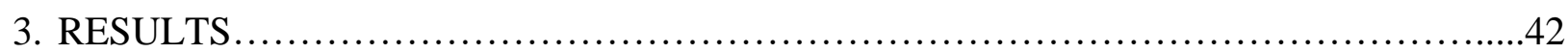

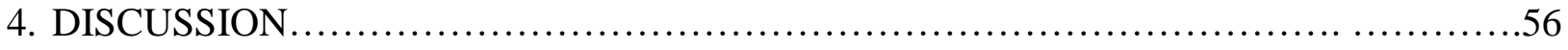

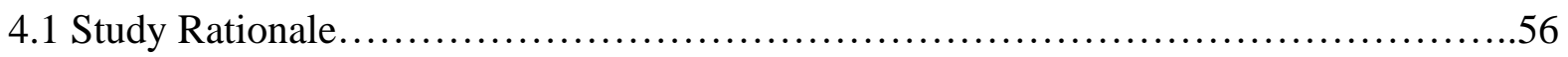

4.2 Summary of Major Findings.................................................... 57

4.2.1 Comparison of EPA-defined dust lead hazard concentration level detection in dust

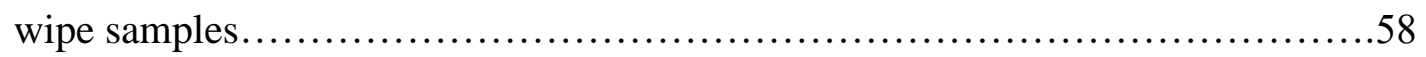

4.2.2 Bland Altman analysis of XRF and ICPMS measurements.....................59

4.3 Comparison of lead concentration in floor and windowsill samples....................60

4.4 Implications of study findings for public health..................................61

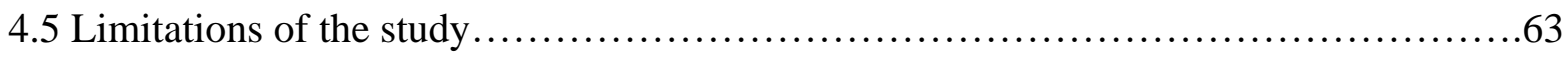

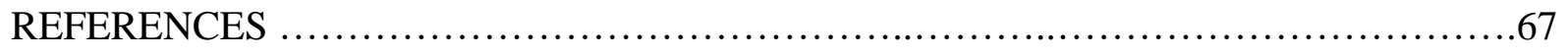

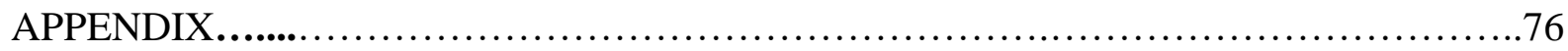

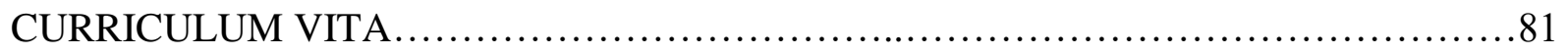




\section{LIST OF TABLES}

Table 1. Frequency distribution table of dust wipe samples collected and sample location....

Table 2. Descriptive statistics for lead concentration in dust wipe samples measured by XRF and

ICPMS

Table 3. Comparison of EPA-defined dust lead hazard concentration level detection in floor dust

wipe samples

Table 4. Comparison of EPA-defined dust lead hazard concentration level in windowsill dust

wipe samples

Table 5. False Negative rates of XRF device......

Table 6. Descriptive statistics for the distribution of differences between ICPMS and XRF

measurements.

Table 7. Summary statistics of Shapiro-Wilk normality test for difference between XRF and

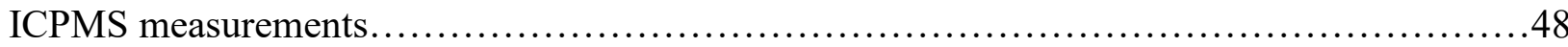

Table 8. Summary of ranks for Wilcoxon signed rank test of XRF and ICPMS values .........49

Table 9. Results of Wilcoxon signed rank test comparing XRF and ICMPS values....

Table 10. Descriptive statistics for lead concentration in floor dust wipe samples measured by

ICPMS.

Table 11. Summary statistics of Shapiro-Wilk normality test for ICPMS measurements of floor dust wipe samples.

Table 12. Descriptive statistics for lead concentration in windowsill dust wipe samples measured by ICPMS .51

Table 13. Summary statistics of Shapiro-Wilk normality test for ICPMS measurements of windowsill dust wipe samples 
Table 14. Summary of ranks for Mann-Whitney U test for floor and windowsill lead

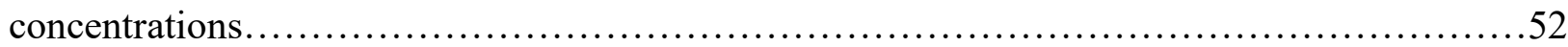

Table 15. Results of Mann-Whitney U test comparing lead concentrations in floor and

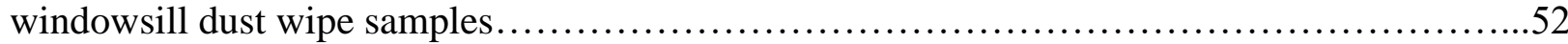




\section{LIST OF FIGURES}

Figure 1: Process of the flame AAS .................................................

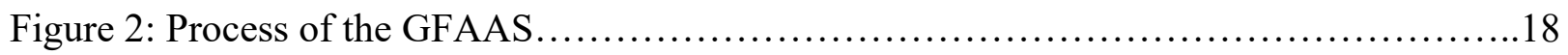

Figure 3. Components of inductivity coupled plasma mass spectroscopy (ICP-MS)...........23

Figure 4. Process of the portable X-ray Fluorescence device............................27

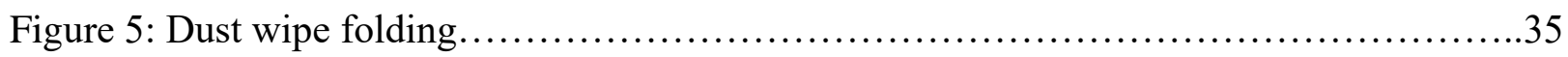

Figure 6: Complete dust wipe surface measurement showing area of each reading............37

Figure 7. Distribution plot of differences between measurements by ICPMS and XRF with

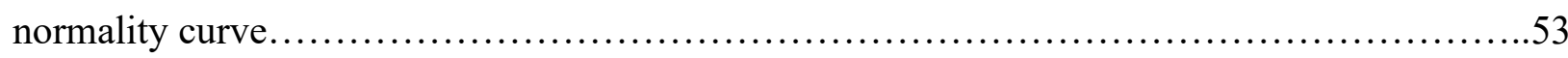

Figure 8. Bland-Altman plot showing the agreement between an XLp 300 XRF device and Agilent 7500cx ICPMS device for analyzing dust wipe samples for lead..................54

Figure 9. Distribution plot for ICPMS measurements of floor dust wipe samples with normality

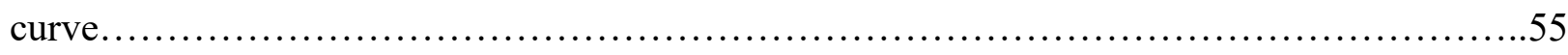

Figure 10. Distribution plot for ICPMS measurements of windowsill dust wipe samples with

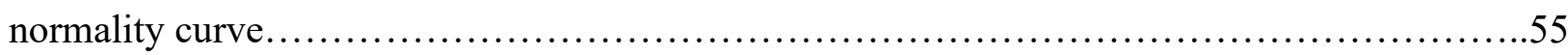




\section{CHAPTER 1 \\ INTRODUCTION}

\subsection{Effects of childhood lead exposure}

Lead is one of the most dangerous environmental toxic substances found in many countries today. Among other effects, it is a neurotoxin and among children, has no safe exposure level. (CDC, 2012, Vorvolakos, Arseniou \& Samakouri, 2016). In both children and adults, at high levels of exposure, lead attacks the brain and central nervous system and can cause convulsions, behavioral problems, and even death. At chronic low levels of exposure during development, lead causes changes in brain and kidney development, disrupts the immune system, lowers IQ scores and diminishes cognitive and motor function (Sobin et al., 2013, Sobin et al., 2015, Skröder et al., 2016).

For example, in a study by Sobin et al. (2015), the effects of early chronic low-level lead exposure on neurobehavioral function (working memory, fine motor dexterity, visual attention, and short-term memory) were assessed in young children between the ages of 5 and 12. It was found that blood lead levels (BLL) predicted levels of working memory, with higher lead levels resulting in poorer working memory. As blood lead levels increased, working memory decreased. The findings of the study provided further evidence that blood lead levels less than $5.0 \mu \mathrm{g} / \mathrm{dl}$ disrupted early neurological function in children

In another study in rural Bangladesh, Skroder et al. (2015) assessed blood pressure and kidney function in preschool-aged children in relation to prenatal lead exposure. Exposure to lead was assessed by measuring the mothers' blood lead level during gestational weeks 14 and 30. Kidney function was assessed by the estimated glomerular filtration rate (eGFR) and by kidney volume. It was concluded that as blood lead levels increase during gestational week 30, 
the kidney volume decreases. The effects were more observed in female as compared to male children

Child lead exposure has broader implications for public health. For example, one study (Lanphear et al. 2018) quantified the relative contribution of environmental lead exposure to allcause mortality, cardiovascular disease mortality, and ischemic heart disease mortality. Results suggested that low-level environmental lead exposure was a significant risk factor for cardiovascular disease mortality. Thus efforts to prevent deaths from cardiovascular disease should include efforts to reduce lead exposure.

\subsection{Trends in child lead exposure}

In 2012, the CDC lowered the reference dose of BLLs in children to $5 \mu \mathrm{g} / \mathrm{dl}$ from the previous $10 \mu \mathrm{g} / \mathrm{dl}$ set in 1990 (CDC, 2019). This change was consistent with a gradual lowering of what was considered an acceptable BLL in children, starting in 1960 with a reference value of $60 \mu \mathrm{g} / \mathrm{dl}$, to $40 \mu \mathrm{g} / \mathrm{dl}$ in 1973 , then $30 \mu \mathrm{g} / \mathrm{dl}$ in 1975 and $25 \mu \mathrm{g} / \mathrm{dl}$ in 1986 (CDC, 1991). The lowering of the standard in 2012 to BLLs $\leq 5 \mu \mathrm{g} / \mathrm{dl}$ was based on a recommendation from the Advisory Committee on Childhood Lead Poisoning Prevention (ACCLPP) to base the reference dose on the $97.5^{\text {th }}$ percentile of the National Health and Nutritional Examination Survey (NHANES) (CDC, 2012). Health risks associated with low-level lead exposure in children have been demonstrated in animal model studies (Sobin et al., 2013; Sobin et al., 2015), and there are calls for the CDC to further lower the current, actionable threshold (Gilbert and Weiss, 2006).

While remarkable progress has been made in reducing the blood lead level (BLL) of children in the U.S., lower-level exposure persists in hundreds of thousands of children nationwide (CDC, 2019; Raymond \& Brown, 2017). For example, non-Hispanic black children, children living in families below the federal poverty line, and children living in older housing 
have a significantly increased risk of higher blood lead levels (EPA, 2017). The child lead exposure crisis in Flint, Michigan, alerted child health experts and the public to the continuing dangers of lead exposure. Investigative journalism conducted by Reuters following the crisis in Flint, Michigan, found that in over 3,800 U.S. cities, at least $10 \%$ of children have BLLs $\geq$ $5 \mu \mathrm{g} / \mathrm{dl}$, which is nearly, double the rates reported in Flint, Michigan (Pell \& Schneyer, 2016).

In February 2018, the U.S Environmental Protection Agency (EPA), together with the president's task force on Environmental Health and Safety Risks to children, discussed steps to develop a federal action plan to reduce childhood lead exposure and eliminate associated health impacts. The EPA released the EPA War on Lead document, which outlined strategies for the federal government to combat lead exposure in U.S children through collaboration with a range of stakeholders, including states, tribes, and local communities, together with businesses, homeowners, and parents (EPA, 2018). One of the four stated goals in the action plan focuses specifically on reducing children's exposure to the major lead sources (EPA, 2018).

\subsection{General sources of lead}

Historically speaking, child lead exposure in the United States has been attributable to four major sources: peeling and chipping lead-based paint, leaded gasoline, lead pipes, and smelting operations. (Dignam et al., 2019).

Many cities in the US preferred lead pipes to iron pipes to distribute water because lead was more malleable and durable than iron (Troesken 2006, Troesken \& Beeson, 2003). The lead pipes corrode over time and contaminate the water running through them with lead causing harmful effects to people who use the water for drinking and cooking (Troesken, 2006). Lead in plumbing was banned in 1986 (Weitzman et al. 2013); however, thousands of homes in the United States still have lead service lines and lead plumbing (Cornwell et al. 2016). Cities with 
lead service lines are required to treat their water to prevent corrosion, in accordance with the EPA's Lead and Copper rule (EPA, 1991). However, a report by the Natural Resources Defense Council (2016) found that thousands of cities were not abiding by the rule as of 2015 (Olson \& Fedinick, 2016). In October 2019, the lead and copper rule was modified to improve the actions that city water authorities must take to reduce lead in drinking water. (EPA, 2019) The changes included improved protocols for identifying lead, expanding sampling, and strengthening treatment requirements to prevent lead exposure. (EPA, 2019)

Lead was first added to gasoline in 1921, despite warnings from the public health service, when scientists at General Motors discovered that tetra-ethyl lead could potentially curb engine knock (Lin-Fu, 1991). By 1936, 90\% of gasoline sold in the US contained lead. Lead continued to be used in gasoline until 1972 when the EPA proposed a phase-out of leaded gasoline because of the interference it caused with the operation of the catalytic converter in automobiles (Needleman, 2000), although the growing recognition of the health effects of lead played a role as well. The first phase-out was completed in 1986, but leaded gasoline remained available in the US until the complete ban in 1990 (Needleman, 2000). The average blood-lead level of children in the US dropped from $16 \mu \mathrm{g} / \mathrm{dl}$ in 1976 to $3.2 \mu \mathrm{g} / \mathrm{dl}$ in 1994, with the initial phase-out and subsequent complete de-leading of gasoline in 1990 (Markowitz \& Rosner, 2013). Nevertheless, there remains legacy contamination in soil across the country from decades of emissions from automobiles and trucks. (Zahran et al. 2013).

Another possible source of lead is from active and non-active secondary lead and other smelting sites (EPA, 2018) whose operations mostly focus on the recovery of lead metal and alloys from scrap, and often, mainly lead-acid batteries. Active lead smelting sites pose a threat to the nearby environment by contaminating the air and soil through emissions and waste 
disposal. Non-active lead smelting sites can still be a source because lead stays in the environment until there is proper remediation of the site (Eckel et al., 2001). Between 1931 and 1964, at least 650 lead smelters operated in the US (Standard metal directory, 1931-1964). By 1969, about 150 of at least 650, were reported to be still active (Smith \& Daley, 1987). Presently in the US, at least 30 active lead smelting plants exist that may emit lead-contaminated waste and continue to contaminate the environment (Eckel et al., 2001). Regardless of the fact that most secondary lead smelting plants across the country have been shut down for years, surrounding areas still contain dangerous amounts of lead. (Elliott \& Frickel 2013). Such is the case of the ASARCO plant in El Paso, Texas, a copper smelter which left many areas in the border region contaminated with lead (Sullivan, 2015). Children are especially susceptible to soil lead because they play outdoors and may ingest contaminated soil because of their frequent hand to mouth behavior. (Health Impact Proj., 2017).

Another common source of child lead contamination is interior and exterior house paint. The use of lead in the manufacture of household paint was banned in 1978, but millions of homes in the US, especially in low-income areas, still have lead paint in both the interior and exterior of these homes sometimes buried under layers of newer paint (Cox et al. 2011). The peeling, chalking, chipping, and cracking of the paint creates dust that can be inhaled or ingested by children living in these households. Lead paint found on chewable surfaces such as windows and windowsills, doors and door frames, stairs, banisters, porches, and railings are also hazards for young children. The children can ingest paint chips from these surfaces, which may contain lead (Weitzman et al. 2013).

Other sources of lead include lead in children's jewelry; lead in food items such as Mexican candy and turmeric; lead in car batteries; lead in glazed pottery; lead in cosmetics such 
as kohl and tiro; and some powdered medicinal folk remedies including Greta, Azarcon, ghasard, pay-loo-ah, and kohl (Muller et al., 2018).

Children are exposed to these sources of lead either from the interiors of their homes or from the external environment, from playing outside, at schools, playgrounds, and parks (EPA, 2017). Analysis of the National Health and Nutrition Examination Survey (NHANES) data and the U.S Department of Housing and Urban Development's (HUD's) American Healthy Homes Survey data, identified household lead hazards as important risk factors for elevated BLLs in children (Dewalt et al., 2015, Caldwell et al., 2017).

\subsection{Lead exposure in the home}

There are many potential household child lead hazard sources, for example, chipping and peeling lead paint on walls and friction or impact surfaces, renovation/repair activities; and tracking lead-contaminated soil into the home by both humans and pets. Any or all of these can result in lead particles collecting in household dust (EPA, 2019). Lead particles from clothing worn at hazardous job sites such as auto repair shops or factories can also settle in household dust. During home renovations, lead dust can be created when lead-based paint or shellac is sanded, scraped, or heated (EPA, 2019). When the home is vacuumed with a non-HEPA filter vacuum or swept, settled lead dust can re-enter the air and resettle on surfaces. (EPA, 2019).

In a study conducted by Lanphear et al. (1998), a pooled analysis of 12 epidemiologic studies conducted over a 15 -year time period $(1982$-1997) was used to estimate the contributions of lead-contaminated house dust and soil to children's blood lead levels. The findings of the study demonstrated a strong relationship between interior dust lead loading and children's blood levels and confirmed the assertion that lead-contaminated house dust is the major source of lead 
exposure for children especially those whose blood levels range from 10 to $25 \mu \mathrm{g} / \mathrm{dl}$ (Lanphear et al. 1998).

\subsection{Importance of lead dust as an indicator of lead hazards in the home}

Research has established that interior dust lead loadings are reliable predictors of a child's blood lead level (Davies et al., 1990; Lanphear et al., 1997, 1998b; Gaitens et al., 2009). Children in homes with higher interior dust lead loadings have been shown to have higher blood lead levels (Dignam et al., 2018; Safruk et al., 2017; Gulson \& Taylor, 2017; Charney et al., 1980; Bornschein et al., 1987; Rabinowitz et al., 1985; Bellinger et al., 1986; Lanphear et al., 1998). For example, in a study conducted by Lanphear et al. (1998), the relationship between the major environmental lead exposure sources (household dust, paint, soil, and water) and lead intake among 183 urban children was estimated. The authors analyzed data from the Rochester Lead-in-Dust study, which was a cross-sectional study involving the analysis of 205 children's blood, household dust, water, soil, and paint for lead. The results of the study showed that, for a dust lead loading of $10 \mu \mathrm{g} / \mathrm{ft}^{2}$, the mean blood lead level of children was $4.1 \mu \mathrm{g} / \mathrm{dl}$, and $3.4 \%$ of the children had a blood lead level exceeding $10 \mu \mathrm{g} / \mathrm{dl}$. Increasing dust lead loading from background levels to $100 \mu \mathrm{g} / \mathrm{ft}^{2}$ was estimated to produce a $15.8 \%$ increase in the percentage of children having a blood lead level exceeding $10 \mu \mathrm{g} / \mathrm{dl}$. In addition, a child's blood lead level is $14 \%$ higher when the dust lead loading on the floor is $40 \mu \mathrm{g} / \mathrm{ft}^{2}$ compared to $20 \mu \mathrm{g} / \mathrm{ft}^{2}$. The data from this study suggested that blood lead levels increased even with a very low increase in the level of lead in the dust (Lanphear et al., 1998).

In another study by Dignam et al., 2018, one main objective was to identify risk factors and sources of lead exposure in children residing in several urban neighborhoods in Philadelphia, Pennsylvania. As part of the study, the researchers used a simple random cross-sectional 
sampling strategy to enroll 104 children, 8 years or younger, living in neighborhoods with a history of the lead-emitting industry. The researchers found that $25 \%$ of households of the participating children screened had an elevated entryway floor dust-lead level, and $14 \%$ had an elevated interior window dust-lead level. The results showed that higher BLLs in children were significantly associated with dust lead content in the home. Children in households with elevated dust lead levels were more likely to have BLLs of $5 \mu \mathrm{g} / \mathrm{dl}$ or more (Dignam et al., 2018). This study further substantiated the importance of lead dust in understanding lead exposure in children.

In a similar study by Safruk et al., (2017), the relationship between lead content in household dust, outdoor soil, tap water, and paint within a household, and the corresponding BLLs in children in that home were examined. As part of the study, BLLs of 118 children under the age of 7 years were measured, and environmental samples were collected from their respective households. The geometric mean BLL for the participants was $1.41 \mu \mathrm{g} / \mathrm{dl}$. The findings from the study indicated a significant positive correlation between BLLs and household dust $(\mathrm{p}<0.05)$, even at low levels of lead exposure. The authors suggested that lead dust on floors and window sills should be kept as low as possible to protect children from lead exposure.

In another study conducted by Charney et al., (1980), the authors hypothesized that leadcontaminated household dust was a major source of lead exposure in 99 children between 18 and 72 months. As part of the study, 40 children with blood lead levels between $40 \mu \mathrm{g} / \mathrm{dl}$ and $70 \mu \mathrm{g} / \mathrm{dl}$ were compared with 50 children in the same city with BLLs $\leq 29 \mu \mathrm{g} / \mathrm{dl}$. The dust in the households of children with BLLS between $40 \mu \mathrm{g} / \mathrm{dl}$ and $70 \mu \mathrm{g} / \mathrm{dl}$ contained significantly more lead than is found in the households of the children with BLLs $\leq 29 \mu \mathrm{g} / \mathrm{dl}(\mathrm{p}=0.005)$. The 
findings of this study provided further evidence to show that household lead dust is a major source of lead exposure in children.

Household dust lead levels are also an important predictor in "clearance testing" of residences. Clearance testing refers to the requirement that a certified Lead Risk Assessor verifies that previous levels of lead in dust have been reduced enough to make the residence safe for dwellers, typically at the conclusion of lead hazard control interventions (US EPA, 2001). This process requires visual inspection and the collection of dust wipe samples from the floors and windowsills in a minimum of 4 rooms in the residence. The dust wipe samples are then analyzed to ensure the dust lead levels meet the applicable clearance standards.

\subsection{EPA Dust Lead Hazard Standards}

Given the association of child lead levels and household dust, the US EPA first established dust lead hazard standards (DLHS) in 2001 (EPA, 2001). These standards included $40 \mu \mathrm{g} / \mathrm{ft}^{2}$ for floors and $250 \mu \mathrm{g} / \mathrm{ft}^{2}$ for window sills and were stated in a final rule entitled, "Identification of dangerous levels of lead," also known as the 2001 LBP Hazards Rule (EPA, 2001). The EPA considered the health implications associated with BLLs that corresponded to various dust lead levels to determine the appropriate standards. The standards were established primarily on the amount of lead dust required to produce a child BLL of $10 \mu \mathrm{g} / \mathrm{dl}$, which was the level of concern recognized by the CDC at the time. The EPA explained that health effects at BLLs lower than $10 \mu \mathrm{g} / \mathrm{dl}$ were not well substantiated (EPA, 2001). The ability to achieve the established dust lead levels as well as economic implications, were also taken into consideration in defining the standards (EPA, 2001).

As more data accumulated showing effects on child development at BLLs lower than $10 \mu \mathrm{g} / \mathrm{dl}$, the EPA issued a policy to establish new and more stringent dust-lead hazard standards 
(DLHS) for lead dust in the home, effective January 2020. The new lead dust hazard action levels are $10 \mu \mathrm{g} / \mathrm{ft}^{2}$ for floors and $100 \mu \mathrm{g} / \mathrm{ft}^{2}$ for window sills and troughs. (EPA, 2019). Further action is required from the EPA to lower the new lead dust hazard action levels (Gilbert \& Weiss, 2006). Toxicological studies have established that the current lead dust hazard action levels are able to produce BLLs higher than $5 \mu \mathrm{g} / \mathrm{dl}$ in children (Gilbert \& Weiss, 2006; American Academy of Pediatrics, 2016). According to a 2016 report by the American Academy of Pediatrics, a more protective standard $\left(5 \mu \mathrm{g} / \mathrm{ft}^{2}\right.$ for floors and $50 \mu \mathrm{g} / \mathrm{ft}^{2}$ for window sills) would reduce the likelihood that an exposed child would have a BLL exceeding the current CDC's action level of $5 \mu \mathrm{g} / \mathrm{dl}$ (American Academy of Pediatrics, 2016).

The recognition of the threats posed by household lead dust has increased the interest of public health professionals, lead risk assessors, and state agencies in inexpensive methods to quickly and effectively determine the existence of lead dust hazards in the home environment.

\subsection{Currently Available Methods for Testing for Lead Dust Hazard Sources in the Home}

\section{Environment}

There are many different approaches available to consumers and professionals for testing the home environment for lead dust hazard sources. The cost, reliability, and validity of each of these methods varies broadly and will be discussed below.

\subsubsection{Home spot lead test kits}

Spot lead test kits for finding potential lead hazard sources in the home have been available since the 1970's (National Institute of Standards, 2000). There are generally two types of spot test kits used for detecting lead in the home. One type contains rhodizonate ions, which turn pink or red when they react with lead ion. The other type is based on the reaction of sulfide 
ions with lead ions; this reaction produces a color change from clear to gray or black (EPA, 1995). In using a spot test kit, the basic procedure is to cut a notch through, or scratch the surface of the paint, then place the reagent solution on that location, then observe qualitatively whether a characteristic color change occurs indicating the presence of a possible lead concentration (EPA, 1995). There are several spot lead test kits available online and in local retail outlets such as Walmart, Costco, and Target. The EPA, however, does not recognize most of these kits as reliable tests of home environmental lead hazards. After September 2010, the EPA recognized a spot lead kit only if it met both the negative and positive response criteria outlined in the EPA rule 40 CFR 745.88c (EPA, 2018). The negative response criterion states that for a paint containing lead $\geq 1.0 \mathrm{mg} / \mathrm{cm}^{2}$ or $0.5 \%$ by weight, the probability of a negative response from a spot test kit must be $\leq 5 \%$ (with $95 \%$ confidence). The positive response criterion states that for paint containing lead $<1.0 \mathrm{mg} / \mathrm{cm}^{2}$ or $0.5 \%$ by weight, the probability that a spot test kit will give a positive response should be $\leq 10 \%$ (with $95 \%$ confidence) (EPA, 2018). To date, no test kit has met both of the performance criteria outlined by the EPA. However, three lead test kits meet the negative response criterion and are recognized by the EPA when used by certified lead inspectors and risk assessors. The EPA-recognized spot lead test kits are $3 \mathrm{M}^{\mathrm{TM}}$ LeadCheck $^{\mathrm{TM}}$, D-Lead ${ }^{\circledR}$, and the Commonwealth of Massachusetts lead test kits (EPA, 2018).

Home spot test kits were designed to detect lead in paint and lead in other consumer products such as jewelry and glazed ceramics (NIST, 2000). The accuracy of the spot test kits has been established only for leaded paint (NIST, 2000; EPA, 1995; ASTM, 1998). For example, in a study conducted by the National Institute of Standards and Technology (NIST) for the U.S. Department of Housing and Urban Development (HUD), eight different brands of home spot lead test kits from different manufacturers were used to conduct more than 3000 tests. The aim 
was to determine the reliability of spot test kits for detecting the presence of lead in household paint. The tests were conducted by certified lead inspectors according to instructions specified by the manufacturer of each test kit. The study showed that 2550 out of $3000(85 \%)$ spot lead test kits gave false-positive results at lead levels less than $1 \mathrm{mg} / \mathrm{cm}^{2}$ (definition of lead-based paint). The spot lead test kits provided a false negative response of less than 5\% (95\% confidence interval) (NIST, 2000). The authors suggested that home spot lead test kits might be useful as negative screens for lead-based paint (NIST, 2000).

Presently, these home spot lead test kits are marketed and advertised as able to detect lead dust levels that are below or exceed the current U.S EPA dust lead standard for floors $\left(40 \mu \mathrm{g} / \mathrm{ft}^{2}\right)$. In fact, some manufacturers claim the kits are able to detect as low as $2 \mu \mathrm{g}$ of lead (Hybrivet, 2006) but do not indicate whether the $2 \mu \mathrm{g}$ level refers to pure lead or lead in a household media (dust or paint). A review of the peer-reviewed literature produced only one study evaluating the accuracy of the home spot test kits in detecting dust lead levels at the EPA's standard for floors $40 \mu \mathrm{g} / \mathrm{ft}^{2}$ (Korfmacer and Dixon, 2007).

In the study by Korfmacer and Dixon (2007), a trained risk assessor collected 200 LeadCheck swab samples side-by-side with standard dust wipe samples. The dust wipes were analyzed using the EPA 7420 method using graphite atomic absorption spectroscopy (GFAAS). Two-thirds of the samples taken produced a negative result according to the LeadCheck swabs when in fact, levels of dust $\geq 40 \mu \mathrm{g} / \mathrm{ft}^{2}$ were actually present. Thus, the LeadCheck swabs produced a false negative rate of $64 \%$ (95\% confidence interval). Thus, the findings of the study showed that these swabs do not reliably detect levels of lead in the dust at or above $40 \mu \mathrm{g} / \mathrm{ft}^{2}$ using published methods under field conditions (Korfmacer and Dixon, 2007). It should be noted that the accuracy of the spot test kits in detecting low-level lead in household dust has not 
been adequately substantiated by independent studies. This poses a significant concern when using home spot lead test kits to identify floor dust lead hazards under the newly proposed EPA standard of $10 \mu \mathrm{g} / \mathrm{ft}^{2}(\mathrm{EPA}, 2019)$.

\section{Advantages of home spot lead test kits}

The rapid home test kits can provide a valuable tool for residents who want to ascertain potential lead hazards in their homes. They provide immediate results, are economical, and are easily available in stores or online. The kits can also be used by people with little to no technical knowledge on lead testing and, as such, are useful for informing homeowners and potential buyers and/or renters to the potential risk of lead in the home. At the same time, they do not provide a complete risk assessment of the home.

\section{Limitations of home spot lead test kits}

There are growing concerns among public health officials and certified lead risk assessors about the potential of spot test kits to mislead residents that use them to screen for potential lead hazards. The concerns include the inability of residents to identify an appropriate test location, and some residents may not be able to use the kits properly. The subjective nature of color change also poses a concern, especially in color-blind individuals (Korfmacer and Dixon, 2007).

Historically, a major barrier to the use of the spot kits is that the results are qualitative and do not provide an indication of the concentration of lead present (EPA, 2019). Because they are not reliable enough to tell the difference between high and low levels of lead, it is difficult to identify if lead dust levels fall below or above the federal standards and, therefore, may or may not require mitigation.

In addition, the spot lead kits may react with some colorimetric reagents to produce the color change that may indicate a nonexistent lead presence (Korfmacer and Dixon, 2007). On 
the other hand, a false positive reading may needlessly push residents and risk assessors into taking further, but unnecessary, investigative actions for the presence of lead.

Moreover, if the swab is unable to make contact with lead in a form that causes the coloration to occur, no observable color change will occur, presenting a false negative reading (Korfmacer and Dixon, 2007). The failure to detect an existing lead hazard may cause residents to believe that their homes are safe. Residents may conduct renovation or remodeling activities that disturb lead hazard sources and generate higher dust lead loadings. For this reason, methods that may result in unnecessary renovation activities should probably be avoided.

Based on the limitations of the home spot lead test kits described above, the EPA and HUD do not recommend their use by either homeowners or certified lead risk assessors to detect lead in dust in the home environment.

\subsubsection{Laboratory tests}

An alternative to home testing kits involves certified lead risk assessors and trained community members collecting dust lead samples according to standardized techniques adopted by the U.S. EPA or the U.S. Department of Housing and Urban Development (HUD). A detailed description of the dust wipe collection can be found in Appendix C. The collected and carefully packaged samples are sent to an accredited laboratory, digested and analyzed by either inductively coupled plasma mass spectrometry (ICPMS) or atomic absorption spectrometry (AAS). These methods are valid and reliable for determining the concentration of lead in different environmental media such as water, soil, paint chips, and dust (Thermo Elemental handbook of elemental analysis, 2001). 


\subsubsection{Atomic Absorption Spectrometry (AAS)}

About 60 years ago, since its development as an analytical technique, atomic absorption spectrometry (AAS) has become a firmly established method for the determination of trace quantities of metals such as mercury, cadmium, arsenic, nickel, chromium, and lead in solutions (Van Loon, 1985). The first commercial AAS instruments that were introduced in the early 1960s used flame procedures for the production of analyte atoms (Van Loon, 1985). In flame atomic absorption spectrometry, either acetylene or a nitrous acetylene flame is used to evaporate the solvent and dissociate the sample into its component atoms (Van Loon, 1985). The technique of the flame atomic absorption spectrometry is illustrated in figure 1.

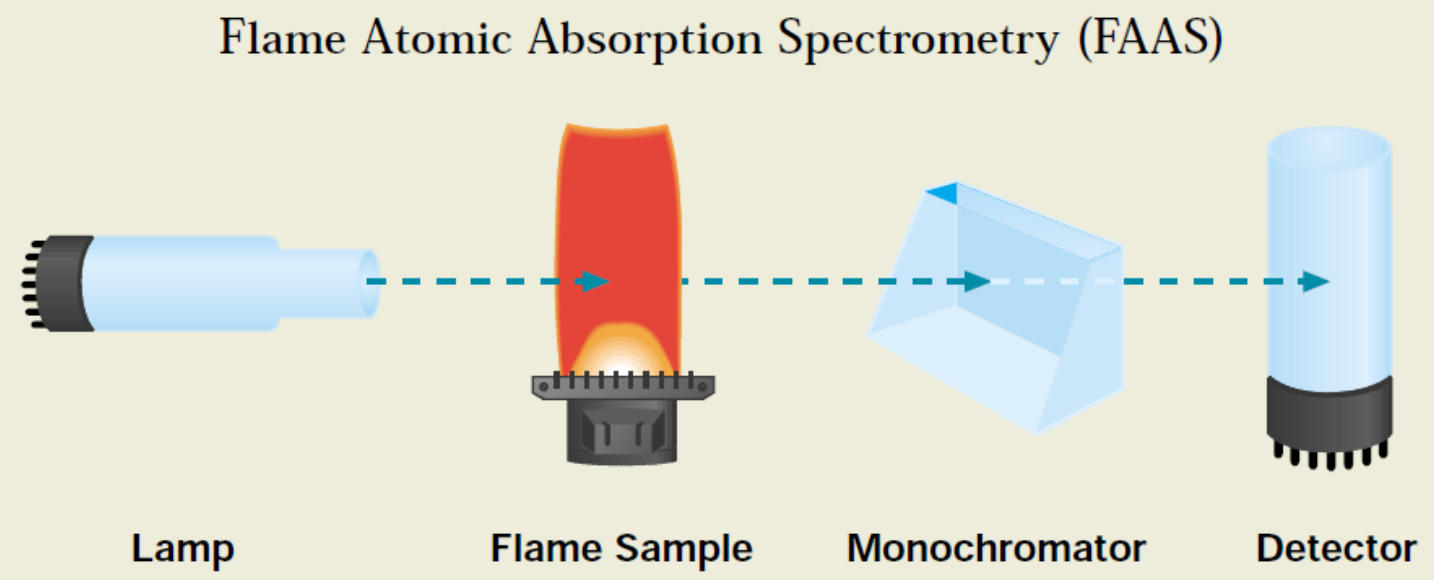

Figure 1: Process of the flame AAS (Thermo Elemental handbook of elemental analysis, 2001)

Briefly, a hollow cathode lamp is selected depending on the element to the determined.

Light from this cathode lamp passes through the cloud of atoms, and the atoms of interest absorb the light from the lamp. The amount of light absorbed is measured by a detector, and this is used to calculate the concentration of that element in the original sample. (Thermo Elemental handbook of elemental analysis, 2001). 
Flame atomic absorption spectrometry may be chosen over other spectrometry techniques because they are usually easy to set up and run, and require minimal operational skill (Butcher, 2017). Method development in flame atomic absorption spectrometry is also very easy, and there is a vast archive of established methods that can be accessed (Butcher, 2017). The compact nature of the instrument also allows for easy handling (Thermo Elemental handbook of elemental analysis, 2001). The flame AAS has low capital and operation costs, which can be beneficial to laboratories with low budgets (Butcher, 2017). Additionally, the flame AAS is the fastest technique when measuring the concentration of a single element in a sample. Only 4 to 5 seconds per measurement are required to obtain a reading (Thermo Elemental handbook of elemental analysis, 2001).

The large sample volume required for flame AAS analysis is a limitation of the technique. For example, the most commonly available equipment requires a volume of $10 \mathrm{ml}$ for analysis. If the sample volume is inadequate, there may not be enough sample to be converted into a conventional flame, aerosol, or mist of tiny droplets, a process known as nebulization, and absorbed by the light from the cathode lamp (Butcher, 2017). This limitation makes flame AAS a less than ideal technique when analyzing samples that may be difficult to get large in large volumes, such as blood. Furthermore, isotopic analysis is not possible when using flame AAS (Butcher, 2017). Isotopic analysis refers to the ability to characterize the primary isotope in a given sample with detectable lead and is important during remediation of lead sources such as lead-contaminated soil. Knowing the exact lead isotope present in samples can inform understanding of the bioaccessibility of a lead contaminant and help to link the contaminant to a specific source or sources (Denys et al., 2007). Also, regarding flame AAS, unattended operation is not possible due to the flammable gas risk. Lastly, although this is not applicable to lead, the 
flame AAS is not ideal for detecting certain metal elements with exceptionally high melting points such as boron (B), vanadium (V), zirconium $(\mathrm{Zr})$, and molybdenum (Mo). This is because the maximum temperature reached in flame $\operatorname{AS}\left(2600^{\circ} \mathrm{C}\right)$ is not enough to break down compounds of these elements (Adams et al., 1988). Analytical sensitivities of the flame atomic absorption spectrometry range from $1 \mu \mathrm{g}$ to $1 \mathrm{mg}$. This is relevant when analyzing samples with a wide range of concentration levels for the same element or different elements (Adams et al., 1988) but problematic for efficiently detecting elements of lower concentration levels (Butcher, 2017).

To achieve greater sensitivity, much effort has focused on improving atomization techniques through the development of non-flame atomizers to replace conventional flame-based techniques (Butcher, 2017). These non-flame atomizers are recommended if the sample size is inadequate to be nebulized (Butcher, 2017). There are different variations of the non-flame atomic absorption spectrometry techniques. These variations depend on the type of atomizer they use. The atomizers can be graphite tubes, rods, and crucibles, or metallic elements such as tungsten, tantalum, and platinum. Of these, the most commonly used are graphite tubes in the Graphite Furnace Atomic Absorption Spectroscopy (GFAAS) technique (Adams et al., 1988). Most laboratories use GFAAS to analyze dust wipe samples for lead and other trace elements and hence will be comprehensively described.

\subsubsection{Graphite Furnace Atomic Absorption Spectroscopy (GFAAS)}

Graphite furnace atomic absorption spectrometry GFAAS also known as electrothermal atomic absorption spectroscopy (ETAAS), is similar to flame AAS, except in GFAAS, the flame is replaced by a small, electrically heated graphite tube, or cuvette, which is heated to a temperature up to $3000^{\circ} \mathrm{C}$ (Welz \& Sterling, 1999). The heated graphite furnace allows the 
thermal energy to break chemical bonds within a sample held in the graphite tube, which then generates the cloud of atoms. (Butcher \& Sneddon, 1998)

All GFAAS instruments have the following basic components: a source of light, usually a lamp that emits radiation; an atomization chamber/graphite tube/cuvette in which the sample is vaporized; and a monochromator for selecting only one of the characteristic wavelengths of the element of interest. There is also a detector, generally a photomultiplier tube that measures the amount of absorption, and a signal processor-computer system. The signal processor-computer system can be a digital display, a printer, or a strip chart recorder. (Butcher, 2017)The technique of the graphite furnace atomic absorption spectrometry is illustrated in figure 2 below.

\section{Graphite Furnace Atomic Absorption Spectrometry (GFAAS)}

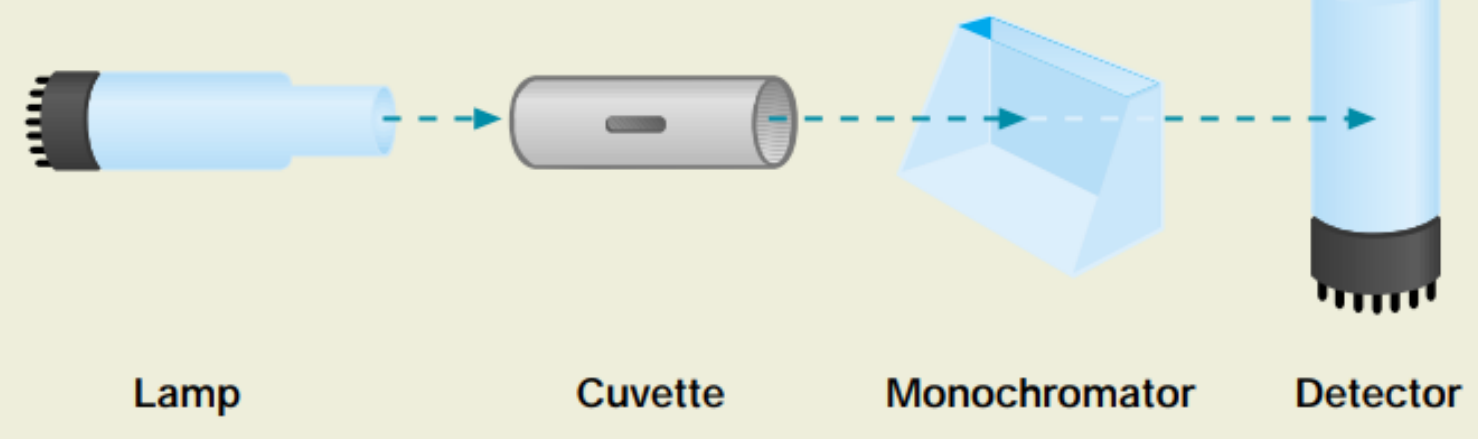

Figure2: Process of the GFAAS (Thermo Elemental handbook of elemental analysis, 2001)

Briefly, the instrument is allowed to warm up after it is powered on and calibrated. The prepared sample is deposited in the graphite tube either manually or through an automated sampler. The sample is vaporized when the graphite tube is heated, and the analyte is atomized. Radiation from the lamp is then directed through the vapor. The atoms absorb this radiation and 
gain higher electronic energy levels. The amount of energy absorbed is proportional to the atomic element concentrations. Concentration measurements are usually determined from a working curve after the GFAAS instrument is calibrated with standards of known concentration (Thermo Elemental handbook of elemental analysis, 2001). The final concentration of an element in a sample is usually the average of triplicate readings (Thermo Elemental handbook of elemental analysis, 2001). Most available GFAAS instruments are fully controlled by a personal computer that uses programmable software (Butcher, 2017). The software makes it possible for a semi-automated GFAAS process. Steps such as calibration and sample dilutions can be done with the automatic software before analysis (Butcher, 2017).

Advantages of GFAAS. GFAAS analysis allows direct solid sample analysis of lead. This has distinct advantages over protocols that involve acid digestion used in Inductively Coupled Plasma (ICP) techniques (Lemaire et al., 2013). For example, direct solid sampling allows a significantly reduced risk of contamination; it also prevents the loss of analytes. Due to the lack of dilution in this protocol, there is a higher sensitivity. Furthermore, the lack of extensive preparation procedures eliminates the use of possibly hazardous and corrosive chemicals (Borges et al., 2006; Lemaire et al., 2013; Welz et al., 2005).

Direct solid sampling is also preferred over acid digestion methods for sample preparation when analyzing materials with complex matrices, such as the determination of lead in lipstick. For instance, in a study to compare the performance of direct solid sampling highresolution continuum source GFAAS to two acid digestion methods (digestion with and without hydrofluoric acid) for sample preparation in inductively coupled plasma mass spectrometry (ICPMS), certified and cosmetic raw material samples were prepared according to the three sample preparation techniques. All three methods obtained good reliability, however only 
ICPMS with hydrofluoric acid sample digestion and GFAAS with direct solid sampling allowed complete recovery of lead. The authors suggested the use of ICPMS with microwave-assisted acid digestion without the use of hydrofluoric acid as another alternative to achieve complete recovery of lead (Lemaire et al., 2013).

The advantages of GFAAS also include the small sample size required for analysis (usually between $20 \mu \mathrm{L}$ and $100 \mu \mathrm{L}$ ) and a minimized interference as compared to FAAS. When using GFAAS rather than FAAS, an increase in sensitivity of between one and three orders of magnitude can be realized along with a very good detection limit for lead. The detection limit or lower limit of detection (defined as the lowest concentration of an element that can be reliably detected by a given instrument) is very important when deciding on a technique to use for analysis of a sample (International Union of pure and applied chemistry (IUPAC, 1997). The detection limit for lead in most samples for GFAAS is $0.04 \mathrm{ppb}$ (Thermo Elemental handbook of elemental analysis, 2001). This detection limit is for the GFAAS technique and does not represent the performance of a particular instrument. Different GFAAS instruments may have different detection limits depending on the manufacturer.

Limitations of GFAAS. The frequently stated major disadvantages of GFAAS approach include slower analysis time, which is typically 1 to 5 minutes per sample (Welz et al., 2005); difficulty in standardizing the protocol within the laboratory; the complex calibration methods required; and the relatively high cost associated with elemental analysis. In addition, isotopic analysis is not possible with GFAAS (Thermo Elemental handbook of elemental analysis, 2001).

Given the disadvantages, there has been a long-standing need to improve on atomic absorption techniques to be able to match the more recent, efficient, and precise inductively 
coupled plasma mass spectrometry (ICPMS) technique (Welz et al., 2005). While earlier AAS methods were suitable for many analyses, the need for improved performance with respect to background correction, wavelength selection, and faster analysis time, are needed to match the rapid multi-elemental capability of ICPMS (Welz et al., 2005). This led to the development of a high-resolution continuum source atomic absorption spectrometry (HR-CS AAS) in the mid1990's (Frientiu et al., 2013), which has made it possible to overcome some of the limitations in atomic absorption spectrometry (Silva et al. 2005; Welz et al., 2007). This instrument, the first of its kind, was designed by a group of researchers from the Institute for Analytical Sciences in Berlin, Germany, led by Becker-Ross (Resano M \& Garcia-Ruiz E., 2011). HR CS AAS provides enhanced flexibility in wavelength selection and permits superior background correction by simultaneous measurement of atomic and background absorption (Resano M \& Garcia-Ruiz E., 2011). In addition, this technique offers greater potential for fast-sequential multi-elemental analysis and allows the use of just one source for all analyses (Resano M \& Garcia-Ruiz E., 2011). This upgrade is commercially available for both flame atomic absorption spectrometry (HR-CS FAAS) and graphite furnace atomic absorption (HR-CS GFAAS). The effectiveness of both variations for analyzing different samples for lead has been demonstrated in several studies (Frientiu et al., 2013; Resano M \& Garcia-Ruiz E., 2011; Welz et al., 2003).

For example, a study was conducted by (Frientiu et al. (2013) to validate high-resolution continuum source flame atomic absorption spectrometry (HR-CS FAAS) for the fast sequential determination of different hazardous metals ( $\mathrm{Ag}, \mathrm{Cd}, \mathrm{Co}, \mathrm{Cr}, \mathrm{Cu}, \mathrm{Ni}, \mathrm{Pb}$, and $\mathrm{Zn}$ ) in soil samples. The study showed that the limits of detection for lead $(1.4 \mathrm{mg} / \mathrm{kg})$ and cadmium $(0.14 \mathrm{mg} / \mathrm{kg})$ in soil samples for the high-resolution continuum source flame atomic absorption spectrometry were close to those in inductively coupled plasma atomic emission spectrometry. $(2.5 \mathrm{mg} / \mathrm{kg}$ and 
$0.05 \mathrm{mg} / \mathrm{kg}$ respectively). In addition, a Bland Altman statistical method showed no statistically significant difference between the HR-CS FAAS and ICPAES (95\% confidence interval). The findings of the study showed that high-resolution continuum source flame atomic absorption spectrometry is effective for the fast sequential multi-elemental determination of hazardous metals in soil (Frientiu et al., 2013).

Despite the many improvements in method development and instrument upgrades in atomic absorption spectrometry (AAS), the inductively coupled plasma mass spectrometry (ICPMS) remains the most precise and effective analysis existing today (Thermo Elemental handbook of elemental analysis, 2001).

\subsubsection{Inductively Coupled Plasma Mass Spectrometry (ICPMS)}

Inductively coupled plasma mass spectrometry (ICP-MS) is the most commonly used method to analyze both biological and inorganic samples to determine metal concentrations because it is capable of analyzing almost all elements in the periodic table and also capable of analyzing solutions, solids, and gases (Allabashi et al., 2009). The ICP-MS is a technique that uses a high-temperature ICP source usually in a range between 6000 and 10000 Kelvin to convert the atoms of the elements in a sample to ions (typically positive ions) which are then separated and detected by a mass spectrometer. A typical configuration of an ICP-MS is shown in Figure 3 below. 


\section{Inductively Coupled Plasma Mass Spectrometry (ICP-MS)}

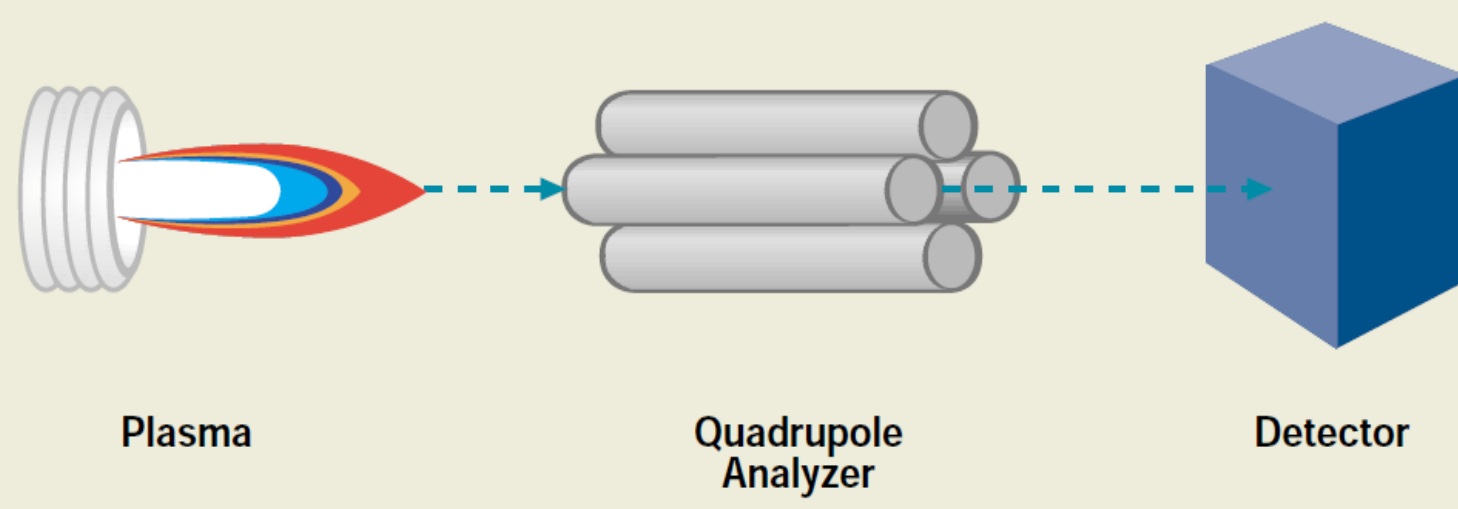

Figure 3. Components of inductivity coupled plasma mass spectroscopy (ICP-MS) (Thermo Elemental handbook of elemental analysis, 2001).

The first step in the ICPMS analysis process is sample preparation (dilution) after digestion. The sample is digested in acid-washed Teflon containers to avoid contamination of samples. The main component needed for dilution is a dilutant, which consists mainly of deionized water with concentrated hydrochloric or nitric acid and Indium and/or Gallium. Usually, 10-500 microliters of sample are added to $5 \mathrm{ml}$ of the dilutant, and the mixture is vortexed to homogeneity (Thermo Elemental handbook of elemental analysis, 2001). The sample is then ready to be introduced to the ICPMS instrument (Thermo Elemental handbook of elemental analysis, 2001).

The sample introduction in ICP-MS can be done using different methods. The easiest method involves the use of analytical nebulizers. Nebulizer types include cross-flow, ultrasonic, desolvating, and pneumatic nebulizers. The completely digested sample is converted into an aerosol by the nebulizer and pumped into the plasma (Beauchemin, 2017). Other sample 
introduction methods include electrothermal vaporization (ETV) (Carey \& Caruso, 1992) and intorch vaporization (Badiei \& Karanassios, 2000). These methods use hot graphite or metal surfaces to vaporize very small amounts of samples to be introduced to the plasma.

After the sample is introduced into the plasma usually made up of argon gas, the plasma's extremely high temperature (between 6000 and 10,000K) causes the sample to separate into individual atoms, a process known as atomization (Beauchemin, 2017). The plasma then ionizes the individual atoms, causing them to lose electrons and become positively charged ions so they can be detected by the spectrometer (Thermo Elemental handbook of elemental analysis, 2001). After ionization, the ions enter a quadrupole or magnetic mass analyzer, where they are separated according to their atomic mass-to-charge ratio. A detector measures the ions, and the concentration of the element of interest is calculated (Thermo Elemental handbook of elemental analysis, 2001).

An alternative to the ICPMS analysis is inductively coupled plasma atomic emission spectrometry (ICPAES) also known as inductively coupled plasma optical emission spectrometry (ICPOES). This technique is similar to the ICPMS technique, however, in ICPAES the individual atoms or ions are excited to a level where they emit light of a characteristic wavelength (Thermo Elemental handbook of elemental analysis, 2001). The intensity of the light emitted is measured by the spectrometer and used to calculate the concentration of the element of interest. Detection limits of ICPAES techniques are usually better than the AAS techniques, however, the ICPMS remains the method with the best detection limits for determination of lead concentrations in a dust wipe sample (Thermo Elemental handbook of elemental analysis, 2001). 
Advantages of ICPMS. ICPMS is an ideal technique for the analysis of a variety of samples, including dust wipes for metal contaminants, because of its multi-element capability. It is capable of simultaneous quantitative analysis of about 100 metals in different media, which makes it very economical when analyzing many samples or testing for many elements (Beauchemin, 2017). Other advantages of using the ICPMS technique include high sensitivity, good precision, large linear dynamic range, the ability to determine the isotope composition of samples, and extremely low detection limits. The detection limit for lead in most ICP-MS instruments is 0.01-0.1parts per trillion (Beauchemin, 2017; Nelms, 2005, Thermo Elemental handbook of elemental analysis, 2001).

Limitations of ICP-MS. ICP-MS has a number of limitations, including the small amount of dissolved solids required to avoid clogging problems. This complicates the analysis by necessitating sample pre-treatment and may involve extra calibration techniques. Other limitations of ICP-MS include the high skill level required for method development and sample preparation, possible exposure to harmful chemicals and gasses, and the high initial capital and running costs required compared to AAS and GFAAS (Thermo Elemental handbook of elemental analysis, 2001).

In sum, all of the described laboratory methods (AAS, GFAAS, and ICPMS) have the capacity to yield valid and reliable measurements of lead in household dust samples. As discussed above, all the methods have some limitations. In addition to the methodologic limitations, individuals require training in sampling techniques to prevent contamination of samples and to ensure quality control during sampling before the samples are sent to the laboratories. Also, analysis reports from laboratories are not readily available and may take up to 2 weeks, depending on the type of service requested. In case there is a child exposed to lead dust 
in the home, the child will continue to be exposed if they remain in the housing or the family may need to temporary relocate to another location, which may present additional costs. In addition, each sample may cost up to $\$ 100$ or an average of around $\$ 400$ per house, excluding shipping and labor costs if a professional is hired.

These limitations of the laboratory methods of dust wipe analysis necessitated the search for an intermediary lead hazard screening method or device that could provide accurate results, perhaps comparable to laboratory methods, but that is also more immediate and cost-effective similar to the home spot lead test kits. The use of the portable X-ray fluorescence device has been touted as a technique that can provide fast, accurate, and precise results regarding the presence of lead hazards in residences (EPA, 2007).

\subsubsection{Portable X-Ray Fluorescence device (pXRF)}

For over 60 years, X-ray fluorescence devices have been used for on-site lead investigations in residential homes to identify lead hazards and assess the risk of child lead exposure (Reece et al., 1972; EPA, 2007). These portable devices use a nondestructive method

of analyzing for lead and other metals in environmental samples including soils, paint chips, and dust wipe samples taken in accordance with HUD and American Society for Testing and Materials (ASTM) methods (HUD,1995; ASTM, 1996). The pXRF device is also capable of testing for lead in consumer products such as jewelry and children's toys (Consumer Product Safety Commission (CSPC), 2015).

Mode of operation of the pXRF. The portable X-ray fluorescence device is used to determine the elemental composition of samples through the use of an X-ray source to excite secondary characteristic x-rays of a sample's constituent elements (ThermoFisher Scientific, 2015). The xray source can be either a sealed radioisotope source or an X-ray tube, and each type has its 
strengths and limitations. For example, the radioisotope source is rugged and proven in-field use, and an x-ray source does not require isotope replacement and allows faster analysis time. The secondary characteristic x-rays emitted are detected and quantified by an x-ray fluorescence spectrometer in the pXRF device (ThermoFisher Scientific, 2015). The technique of the portable X-ray is illustrated in figure 4 below.

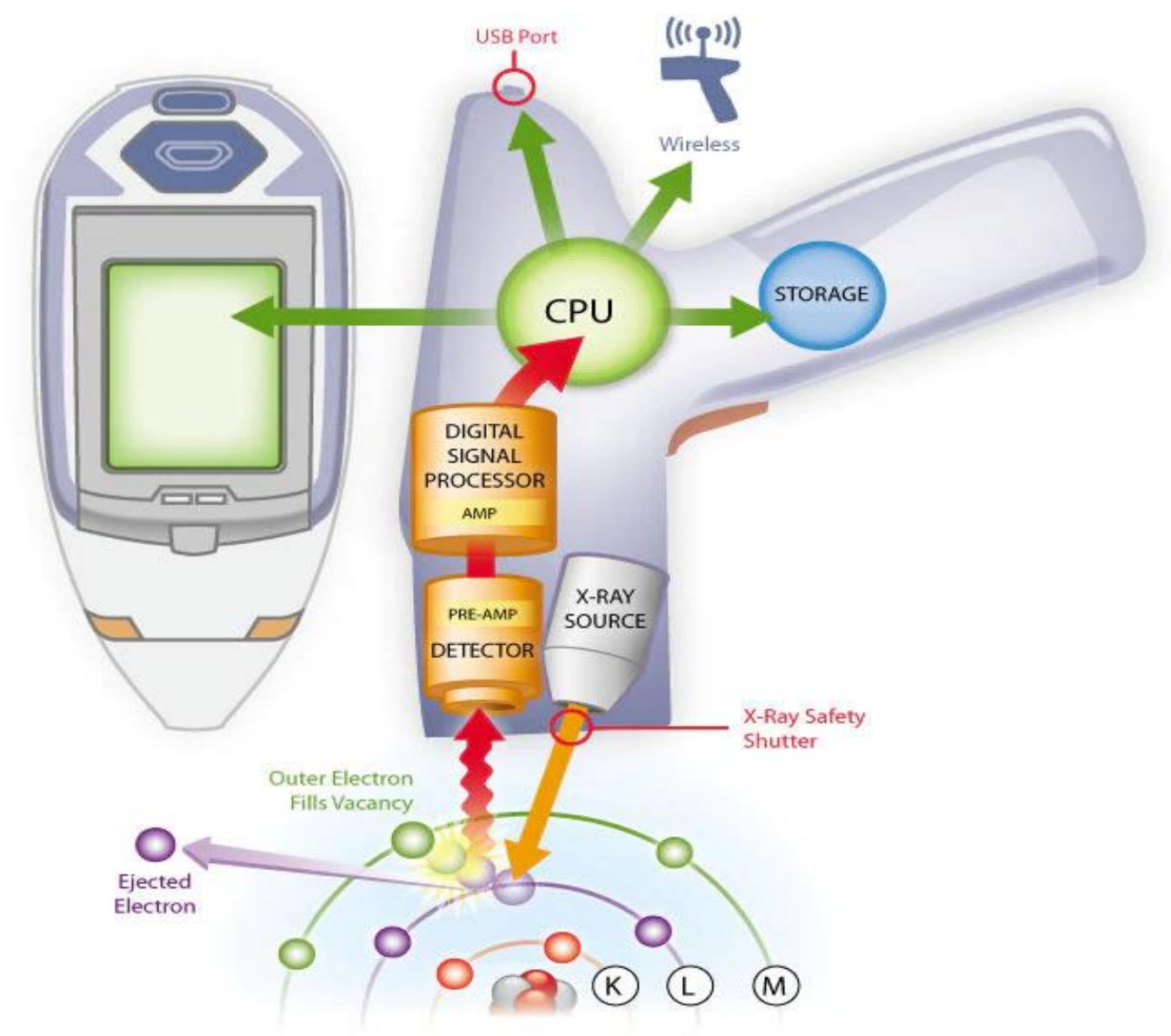

Figure 4. Process of the portable X-ray Fluorescence device (ThermoFisher Scientific Technology focus: X-ray Fluorescence (XRF) in Mining, 2015)

The X-ray Fluorescence Process. The sample is illuminated with primary high energy x-rays from a sealed radioisotope source or x-ray tube energy source. These primary x-rays after striking the sample, are either scattered or absorbed depending on the difference between the 
energy from the x-ray and the binding energy of the atom's K or L shell. The atoms in the sample absorb the energy when the energy from the $\mathrm{x}$-ray is higher than the binding energy in the shells of the atom in a process known as the photoelectric effect (EPA, 2007; ThermoFisher Scientific, 2015). When the atom absorbs the energy from the x-ray, electrons from one of the atom's inner orbital shells are displaced. In an attempt to regain stability, electrons from the atom's outer shells fill the vacancy left by the displaced electrons. The electrons from the atom's outer shells have higher energy states, and they drop to lower energy states to be able to fill the space created by the dislodged electrons. The emission of energy from the electron to achieve a lower energy state is termed as x-ray fluorescence, and the measurement of this energy loss is the basis of XRF analysis (EPA, 2007; ThermoFisher Scientific, 2015). The detector in the $\mathrm{pXRF}$ device converts this energy into electric pulses with heights proportional to the energy of the x-rays. The peak X-ray fluorescence signal at a specific wavelength or energy determines the presence of an element, and the number of counts (heights and/or area of peak) at a given energy per unit of time corresponds to the concentration of the element in the sample (EPA, 2007).

Advantages of the portable X-Ray Fluorescence technique. The portable X-ray Fluorescence device has been approved by several recognized agencies (e.g., EPA, 2007; NIOSH, 1998) for use in analyzing environmental samples for lead because it is simple, fast, and requires no sample preparation. As opposed to ICPMS and GFAAS, XRF analyses are done directly on samples without the need for sample digestion or dissolution, and usually, no chemical waste is generated (ThermoFisher Scientific, 2015). In addition, the XRF technique is nondestructive, and the samples can later be analyzed using laboratory techniques such as GFAAS or ICPMS for confirmation or further analysis (EPA, 2007). The pXRF technique is a very cost-effective 
method for analyzing environmental samples for lead considering the relatively small initial capital needed to purchase the XRF device and the absence of expensive acids and gasses in the analysis process that is required in GFAAS and ICPMS (EPA, 2007; Beauchemin, 2017). Most portable XRF devices can simultaneously detect up to 25 elements in a sample, significantly reducing the time required for sample characterization (ThermoFisher Scientific, 2015). Another advantage of the pXRF technique is the capability of on-site analysis, which allows for immediate lead hazard detection and characterization (EPA, 2007).

\section{Limitations of the portable X-Ray Fluorescence technique. Alongside the X-ray}

fluorescence technology's inability to measure radiation from all elements, radiation exposure concerns for operators is also a limitation of the technique (EPA, 2007; ThermoFisher Scientific, 2015). Interference from other elements with identical fluorescence lines during Xray fluorescence is also a limitation of the XRF technique (Schatzlein et al, 2003). For example, arsenic levels in a sample may make it difficult to determine correct lead levels during XRF measurements (Schatzlein et al, 2003).

\subsubsection{Comparison of pXRF and laboratory techniques for analyzing environmental samples}

\section{for lead}

Several studies and official guidelines have established the accuracy of portable X-ray fluorescence devices for the determination of lead in paint and soil. This is due to recent improvements in portable XRF technology that have increased the sensitivity of XRF measurements (Clark et al., 1999; Markey et al., 2008; Reames G \& Lance L, 2002; EPA, 2007; Cheng-Mau et al., 2012; HUD, 1995; EPA, 1995; EPA, 2007; Muller et al., 2014)

For example, in a study by Chen et al. (2012), the reliability and accuracy of the X-ray fluorescence technique was assessed by comparing the results of XRF in-situ testing with the 
results of inductively coupled plasma atomic emission spectrometer (ICP-AES) analysis of samples. In this study, the portable XRF analyzer was used to estimate the concentration levels of eight heavy metal elements ( $\mathrm{Ni}, \mathrm{Cu}, \mathrm{Zn}, \mathrm{Pb}, \mathrm{Cd}, \mathrm{Cr}, \mathrm{Hg}$, and $\mathrm{As}$.) in 60 in-situ sampling locations. Soil samples taken from these locations were digested using aqua regia acid and analyzed by ICP-AES, and then pairwise comparisons were made between the XRF and ICPAES results. The reliability of the XRF measurements was highest in Lead with relative proximity of 85.17\%; this means that controlling only the values over the pollution threshold limit of $2000 \mathrm{mg} / \mathrm{kg}$, more than two-thirds of soil pollution was captured by the XRF site screening. The results presented in this study suggested that the use of XRF testing is reliable as a screening technique for lead (Cheng-Mau et al., 2012).

Another study was conducted by the Childhood Lead Poisoning Prevention branch of the California Department of Health Services to evaluate a NITON pXRF device for testing soil lead during environmental assessments of lead-poisoned homes with children. As part of the study, the XRF was used to test 119 testing locations in soil from 11 single-family homes built in the 1940s and 1950s. Composite samples were collected from these locations, and the samples were homogenized and retested in the bag with the pXRF. These samples were then analyzed for lead with FAAS. The results of the study showed a significant correlation between pXRF and FAAS values $(\mathrm{R}=0.93, \mathrm{p}<0.0005)$. The authors suggested that the $\mathrm{pXRF}$ device met the criteria for an acceptable screening method for lead in soil (Reames G \& Lance L, 2002).

In two studies conducted to determine the feasibility of using the field-portable XRF analyzer for analysis of lead in soil samples, soil samples were collected from residential areas and industrial regions and sieved to less than 125 micrometers. The authors (Clark et al., 1999 and Markey et al., 2008) found a strong positive correlation between the portable X-ray 
fluorescence device and atomic absorption spectrometry analysis for soil samples sieved to less than 125 micrometers ( $\mathrm{R}^{2}$ values of 0.997 and 0.992 respectively) (Clark et al., 1999; Markey et al., 2008)

In relation to XRF testing for lead paint, a study was conducted by Muller et al. (2014) to evaluate the performance of the pXRF instrument for measuring the lead content of paint in field samples. The authors measured lead content in painted building blocks made of concrete, plaster, or wood with three different pXRF instruments but identical types and compared the results to inductively coupled plasma mass spectrometry (ICPMS) analysis data from paint chips collected from the XRF testing locations. The findings of the study showed that the portable XRF device that emits enough energy for lead $\mathrm{K}$ and $\mathrm{L}$ shell excitation had an accuracy rate of $83 \%$ (19 out of 23 readings) and provided no false-negative measurements. The authors concluded that portable XRF devices that emit sufficient energy for lead $\mathrm{K}$ and $\mathrm{L}$ shell fluorescence appeared to be the most precise and accurate (Muller et al., 2014).

In conclusion, the above described studies and guidelines have demonstrated that the portable XRF device is useful for the determination of lead in paint and soil. However, there are limited studies validating the portable X-ray fluorescence device against more accurate and precise laboratory analytical methods for analyzing lead in dust wipe samples (Sterling et al., 2000; EPA, 2002; EPA, 2003; Harper et al., 2002; Rogers et al., 2012)

For example, the EPA conducted a verification study in 2002 to evaluate the portable $\mathrm{XRF}$ device as an accurate and precise technique to measure lead in dust wipes (EPA, 2003). As part of the study, the vendors of the XLt-700 XRF device analyzed 160 dust wipe samples containing lead concentration between $\leq 2 \mu \mathrm{g}$ to $1,500 \mu \mathrm{g}$. The dust wipe samples included samples prepared from the Environmental Lead Proficiency Analytical Testing Program 
(ELPAT) and the University of Cincinnati (UC) samples prepared from NIST standard reference materials. The samples were then analyzed at an NLAAP accredited lab using Inductively Coupled Plasma Atomic Emission Spectrometry (ICPAES). The reporting limit of the ICPAES instrument and XRF device were $20 \mu \mathrm{g} /$ wipe and $10 \mu \mathrm{g} /$ wipe, respectively. The authors found that for samples with a lead concentration above $20 \mu \mathrm{g}$, there was a strong correlation between XRF results and ICPAES results $(\mathrm{R}=0.999)$. The XRF device also reported nine (9) out of a possible forty-nine (49) false-positive results (reports a concentration above the clearance level of $40 \mu \mathrm{g} / \mathrm{ft}^{2}$ or $250 \mu \mathrm{g} / \mathrm{ft}^{2}$ when the true concentration is below) compared to two (2) from ICPAES instrument. Additionally, the XRF device reported eighteen (18) out of a possible fifty-one falsenegative results (reports a concentration below the clearance level of $40 \mu \mathrm{g} / \mathrm{ft}^{2}$ or $250 \mu \mathrm{g} / \mathrm{ft}^{2}$ when the true concentration is above). The authors concluded that the $\mathrm{pXRF}$ device is slightly negatively biased but within acceptable limits, precise and in good linear agreement with ICPAES results. (EPA, 2003)

In another study by Sterling et al. (2000), the authors tested 185 dust wipe samples collected in the field with a portable X-ray fluorescence (XRF) device and subsequently analyzed by flame atomic absorption spectrophotometry (FAAS) following digestion of the samples. The reporting limits for FAAS and the XRF were $25 \mu \mathrm{g}$ and $20 \mu \mathrm{g}$, respectively. Paired comparisons between the XRF and FAAS results showed no statistical difference $(\mathrm{P}=0.272)$ and a positive correlation of 0.93 for samples that did not contain paint chips. The authors concluded that the XRF could be an excellent method for rapid on-site evaluation of dust wipes for clearance and risk assessment purposes (Sterling et al., 2000).

Though the studies described sought to validate the portable XRF device as an accurate and precise technique to measure lead content in dust wipes, the comparison was against either 
FAAS or ICPAES results, which are less sensitive than ICPMS. More importantly, the experimental design of these studies was particularly focused on the former dust lead hazard standards set by the EPA, which were $40 \mu \mathrm{g} / \mathrm{ft}^{2}$ for floors $250 \mu \mathrm{g} / \mathrm{ft}^{2}$ for window sills. More studies need to be conducted to validate the pXRF device against the currently most precise and accurate analytical method (ICPMS) for analyzing lead in field dust wipe samples taking into consideration the new EPA dust lead hazard standards level limits of $10 \mu \mathrm{g} / \mathrm{ft}^{2}$ for floors $100 \mu \mathrm{g} / \mathrm{ft}^{2}$ for window sills and window troughs. Moreover, it is critical to evaluate not only the correlation of results but the extent to which the absolute values obtained with each method align.

\subsection{Study Aims}

The goal of this study was to test the comparability of the pXRF (portable X-Ray Fluorescence) device to ICPMS (Inductively Coupled Plasma Mass Spectrometry) analyses for detecting lead concentration in household dust samples. ICPMS is the current "gold standard" method for determining the lead concentration in a range of media (e.g., blood, paint chips, soil, and water). While extremely precise and reliable, ICPMS requires specialized laboratory analysis, which can delay the determination of lead hazard sources in homes with lead-exposed children. Determining the comparability of $\mathrm{pXRF}$ readings of dust wipe samples to ICPMS analysis of the same samples could eventually help to inform timely and efficient future recommended methods for assessing critical child lead risks in the home environment. 


\section{CHAPTER 2}

\section{METHODS}

\subsection{Sample Collection}

Dust wipe samples from 10 households were used for this study. A minimum of 8 dust wipes samples per home were collected, yielding a total minimum sample size of 100 samples. Lead dust sampling wipes (Environmental Monitoring Systems Inc., North Charleston, SC) were used to collect samples. The wipes were individually wrapped $5 \times 7.75$ in wipes that meet all ASTM E1792 specifications for sampling materials for lead in surface dust and OSHA Methods ID-125G. The dust wipe samples were collected from carpeted or uncarpeted floors, and window components, , in accordance with a modified version of guidance statement ATSM D7659-10, which provides standards for the sampling of metals and metalloids on surfaces for subsequent analysis using methods such as atomic spectrometry, mass spectrometry, X-ray fluorescence, or molecular fluorescence. Templates were used to collect samples at each location within each room. Clean cardboard templates with inside dimensions of $12 \times 12$ in (Environmental Monitoring Systems Inc., North Charleston, SC) were used for the collection of floor samples.

For areas where the template could not be used and the total sampling area less than $1 \mathrm{ft}^{2}$, such as on window sills, the entire area was wiped. The wiped area was measured and recorded on a sample collection form immediately after the area had been wiped.

For each of the 10 households, blank samples were prepared in accordance with HUD guidelines for dust wipe sampling and analysis. From each house, a minimum of 2 blank samples were prepared (dust wipes that were not wiped on any surface). The wipes were folded to the recommended specifications and placed in a plastic bag similar to the field samples.

\subsection{Sample Preparation}


Following the recommendation for dust wipe testing by XRF (ThermoFisher, Tewksbury, MA), the wipe to be analyzed was folded five times to yield a rectangle approximately 1 in $X 1.5$ in. This is illustrated in figure (5) below.
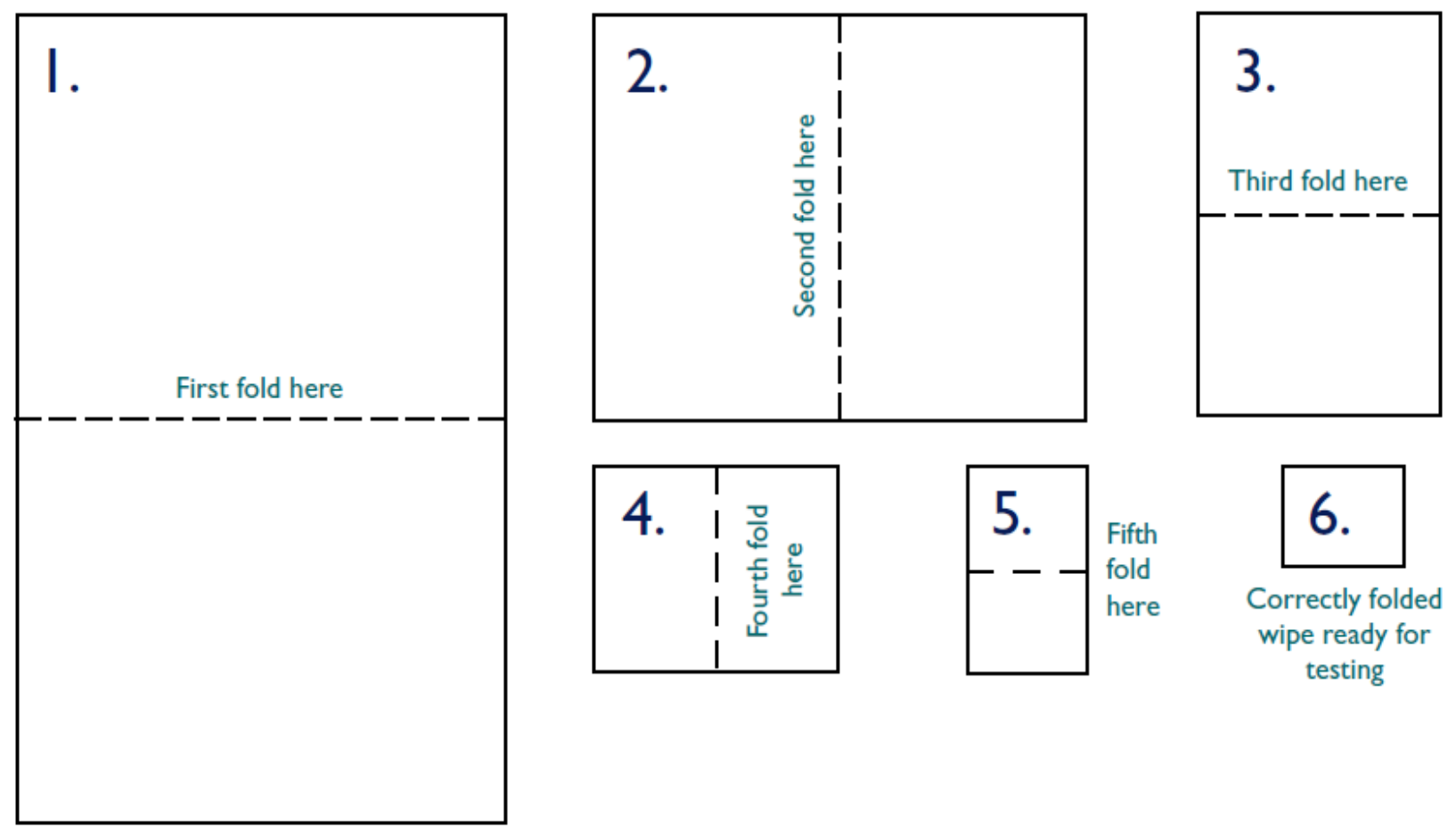

Figure 5: Dust wipe folding (NITON application bulletin: Data quality in Lead dust wipe measurement, 2002)

Each wipe sample was then placed into a $1.5 \times 2$ in low-density polyethylene (LDPE) plastic bag. This packaging maintains the wipe in the desired folded shape with recommended dimensions for the subsequent XRF analysis. A fresh bag was used for each sample to eliminate the potential for cross-contamination of samples.

Following the XRF manufacturers guidelines, each sample was left overnight at ambient room temperature to dry thoroughly prior to testing. (This improves the accuracy and precision of the dust wipe measurements; moisture in the sample will give readings that are lower than the actual 
value). After drying, the wipe in its plastic bag was positioned within the frame of the metal dust wipe holder to be analyzed.

\subsection{Sample Analysis}

All wipes underwent two types of analysis, first using the pXRF device, and then using ICPMS as described below

\subsubsection{Portable XRF Analyses of Samples}

The Niton XLp 300 analyzer (ThermoFisher Scientific, Tewksbury, MA) measures the spectrum and intensity of characteristic fluorescent x-rays emitted by lead in the sample of interest when it is illuminated by gamma rays from a radioactive Cadmium isotope. A combination of the XRF manufacturer's instructions for analyzing dust wipes in thin sample mode and United States EPA method for testing lead in dust wipe using X-Ray fluorescence technology was followed for the testing.

This protocol included an internal self-check by the unit and a self-calibration before taking any measurements. The XRF device performed a self-check on start-up after logging in. After the time and date had been verified, calibration was performed by locating the calibration tab under the "utilities" tab from the main menu. A reading was displayed on the screen after calibration was complete. After self-calibration was completed, calibration verification was performed by testing NIST traceable standards with known lead concentrations. The selfcalibration process was conducted before each use and every 4 hours during operation.

Eight sample measurements per dust wipe were taken from both sides of the wipe to ensure that the entire area of the folded dust wipe sample was properly measured by the spectrometer, and the average of the side with the highest total reading was recorded as the final lead concentration per wipe. Each of the eight sample measurements was taken from a different 
sample orientation for a minimum of 60 seconds. Each time the XRF instrument was positioned in the nose cone adaptor to take a reading of a dust wipe sample, it made contact with a quarter of one side of the dust wipe. Four measurements were taken from the "front" side of the wipe to ensure that the analyzer measured as much of the entire area of that side. This is illustrated in figure 5 below.

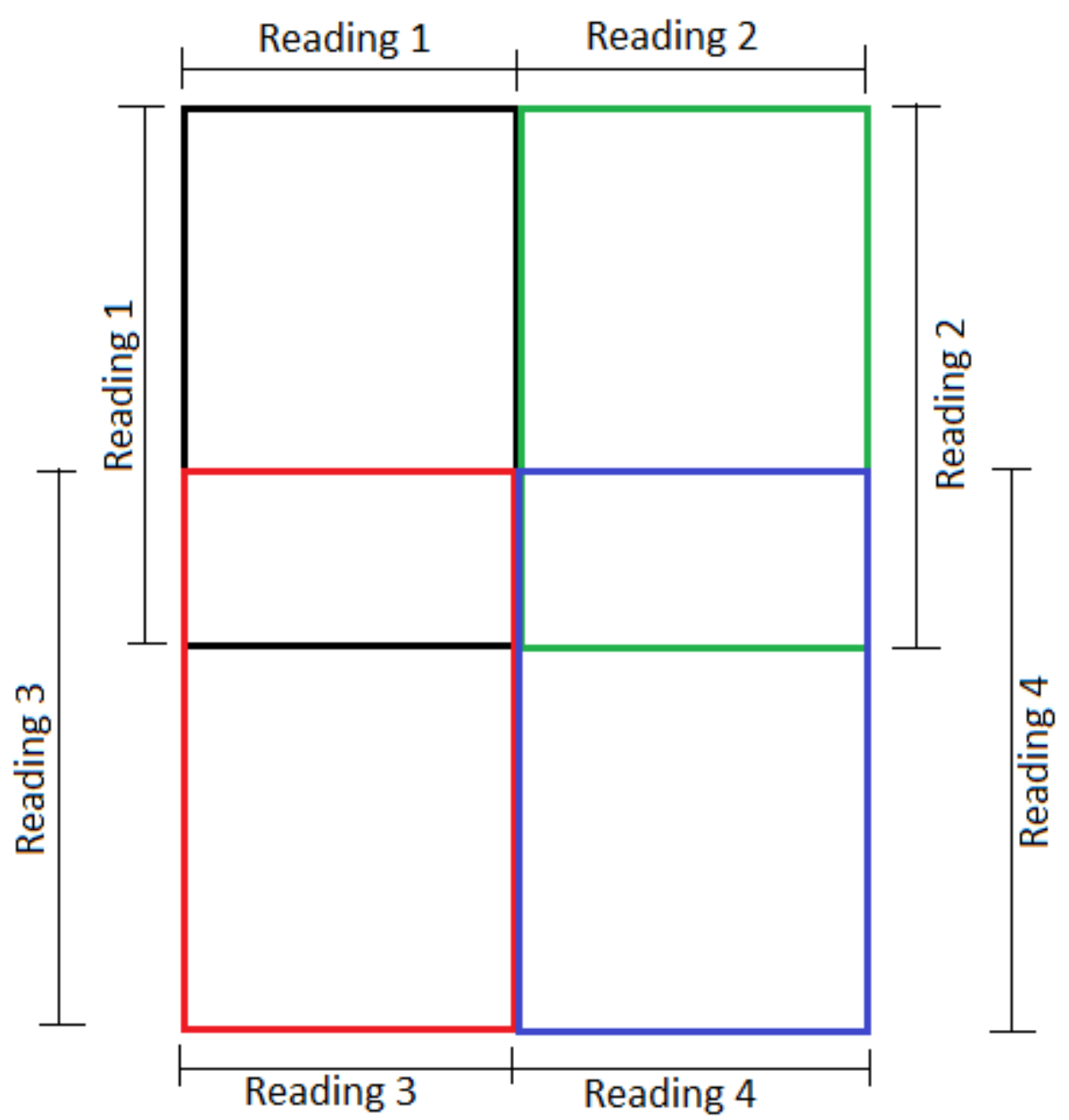

Figure (6): Complete dust wipe surface measurement showing area of each reading.

The measurement screen continually updated during each reading. When the measurement was terminated, the XRF screen updated one final time, and the results were stored in the device for future review. After the fourth measurement was completed, the device automatically averaged the results, and this represented the lead concentration for the front side 
of the wipe. This value was recorded. The wipe was then flipped over, and the protocol was repeated to obtain four measurements on the "back" side of the wipe. The average lead concentrations from the two sides were compared, and the highest average was selected to represent the final lead concentration of the wipe. Because the $\mathrm{pXRF}$ device reads deeper than the total depth of the wipe, this approach was adopted to avoid "double-counting" the total lead concentration. The result was divided by the area wiped to get the amount of lead in $\operatorname{micrograms} / f t^{2}$.

Quality Control. A minimum of 2 field Blank samples were collected in each home visited and treated as experimental samples in all aspects including exposure to the sampling site conditions, drying, storage, XRF analytical procedures and shipping to KSU Soil Chemistry laboratory for digestion (hot plate digestion method, ASTM E1644-17) and ICPMS (inductively coupled plasma-mass spectrometry, Agilent $7500 \mathrm{cx}$ ) analyses. This is to determine if method analytes or other interferences are present in field environment (EPA, 2002).

Two National Institute of Standard Testing (NIST) traceable standards with known lead concentrations were analyzed with XRF device to check the accuracy of the calibration. The standards (dust wipe system 180-037b and 180-038b with lead concentrations of 32-48 $\mu \mathrm{g}$ and 161-242 $\mu$ g respectively, from Thermo Scientific, Niton) were analyzed exactly like the study samples. The two NIST standards were analyzed at the beginning of XRF analysis and after every 4 hours of using the XRF device to the check accuracy of calibration according to manufacturer's instructions.

\subsubsection{ICPMS Analyses of Samples}

This procedure follows the ATSM E1644-17 method, which describes the standard practice for hot plate digestion of dust wipe samples for the determination of lead. The procedure 
covers acid digestion of dust wipe samples and associated quality control (QC) samples for the determination of lead. The procedure in this protocol is based on NIOSH 7082, and NIOSH 7105 and EPA SW-846 Method 3050 - Method Collection for the preparation and analysis of metals in environmental matrices (EPA 1996).

The ICP was calibrated according to the manufacturer's recommendations. (EPA 1994). Standards in the range of $0.1 \mu \mathrm{g} / \mathrm{L}$ to $50 \mu \mathrm{g} / \mathrm{L}$ were analyzed each day of analysis. A check standard was run every ten samples, and recoveries were checked with at least two spiked media blanks per ten samples.

The ICPMS analysis method began with a hot plate/nitric acid digestion of the dust wipes. To summarize, the wipe was digested using a hotplate type heating with $25 \mathrm{~mL}$ of nitric acid and hydrogen peroxide to reach a temperature between 85 to $100^{\circ} \mathrm{C}$. The digestate was diluted to a final volume with distilled water and analyzed by ICPMS.

Quality Control. The field blank dust wipe samples analyzed with XRF device were subsequently analyzed using ICPMS to determine if method analytes or other interferences were present in field environment.

Also, unopened wipes from the same batch of dust wipe samples used were also shipped with the samples collected from the field for ICPMS analysis. These wipes were used as laboratory reagent blanks and are treated exactly as the other samples including exposure to all glassware, equipment, solvents, reagents ad internal standards used for other samples. This was to determine if method analytes or other interferences are present in the laboratory environment, reagents, or apparatus used (EPA, 1994).

Spiked samples were also analyzed with the ICPMS for quality control purposes. These are laboratory samples made from adding a solution of method analytes of known concentrations 
to blank wipes and analyzed exactly like the other samples. This was used to check the accuracy of the laboratory instrument (EPA, 2004). For this study, at least two spiked samples prepared from NIST 2711a certified reference material (CRM) were included with each batch of dust wipe samples analyzed. To prepare the spiked samples, different amounts of the NIST 2711a CRM $(0.1 \mathrm{~g}, 0.2 \mathrm{~g}, 0.3 \mathrm{~g}$ or $0.4 \mathrm{~g})$ were spread onto blank wipes individually. These served as spiked samples used for quality control during ICPMS analyses.

\subsection{Statistical Analysis}

Prior to statistical analysis, all data and distributions were examined. Descriptive analyses were conducted to determine the means and distributions of the pXRF and ICPMS variables. The data from the two sources were then analyzed using the Bland-Altman analysis. This method was selected because, unlike correlation, Bland-Altman analysis was designed to assess the comparability between methods rather than their differences or linear relationship.

\subsubsection{Bland Altman Analysis}

A Bland Altman plot showing the unit differences and averages was prepared, examined, and interpreted. The difference of the two paired measurements (y-axis) was plotted against the mean of the two measurements (x-axis) to determine the degree of agreement between the two methods. The data showing the differences between measurements was assessed for normality, and the limits of agreement was calculated using the mean difference and standard deviation of the mean difference.

\section{A Priori Limits of Agreement}

The acceptable limits of agreement between the two measurements were set before analyses were conducted. Because this approach had not previously been used, the recommended acceptable limits of agreement by Bland Altman was used to assess the comparability of pXRF 
and ICPMS methods. Bland and Altman (1986) recommended that $95 \%$ of data points on the Bland Altman plot must fall within the established lower and upper limits of agreement, or \pm 2 SDs of the mean difference, to establish an acceptable agreement between the two methods.

Further exploratory analyses were conducted to generate hypothesis for future studies on children's exposure to lead hazards in the home. 


\section{CHAPTER 3}

\section{RESULTS}

A total of 109 dust wipe samples were collected from 13 homes. Seventy-six (69.72\%) dust wipe samples were collected from floors; 33 (30.28\%) dust wipe samples were collected from windowsills (Table 1). All samples were analyzed using pXRF and ICPMS (see pages 3438 above).

Prepared samples were first analyzed with the non-destructive pXRF method by taking 4 readings on each side as shown in Figure 6. The pXRF lead concentrations determined for each wipe are presented in Appendix A. Same-side lead concentrations were averaged; the averages were compared, and the highest average was selected to represent the final lead concentration of the wipe. (See pages 36-38 for detailed methods.)

The same samples were then analyzed using ICPMS. The ICPMS lead concentration results determined for each wipe are also presented in Appendix A. Two samples had lead concentrations below the detection limit of ICPMS and were not included in data analyses, leaving a total of 107 floor and window samples.

Table 2 shows the descriptive statistics for 107 dust wipe samples. The skewness metric for XRF (5.383) and for ICPMS (5.068) showed that the data from each method were positively skewed. The skewed distributions are evident from the fact that the medians are much lower than the means for both XRF and ICPMS measurements. Also, there is a high variability between lead concentrations in the dust wipe samples collected with over $70 \%$ of dust wipe samples having

lead concentrations $<10 \mu \mathrm{g} / \mathrm{ft}^{2}$ and less than $8 \%$ of samples having lead concentrations $>300 \mu \mathrm{g} / \mathrm{ft}^{2}$ (Appendix A). 
As shown in Table 2, the median lead concentration determined by XRF was $5.30 \mu \mathrm{g} / \mathrm{ft}^{2}$, as compared to $2.38 \mu \mathrm{g} / \mathrm{ft}^{2}$ determined by ICPMS. (The mean lead concentration determined by XRF was $210.09 \mu \mathrm{g} / \mathrm{ft}^{2}$ as compared to $276.67 \mu \mathrm{g} / \mathrm{ft}^{2}$ determined by ICPMS). The maximum lead concentrations measured were $6083.35 \mu \mathrm{g} / \mathrm{ft}^{2}$ and $7173.82 \mu \mathrm{g} / \mathrm{ft}^{2}$ for XRF and ICPMS, respectively.

Tables 3 and 4 provide a descriptive comparison of the numbers and percentages of samples by each method that detected EPA Dust Lead Hazard levels (> $10 \mu \mathrm{g} / \mathrm{ft}^{2}$ for floors; > $100 \mu \mathrm{g} / \mathrm{ft}^{2}$ for windowsills).

Overall, ICPMS appeared to detect a greater number of samples with lead concentrations above the current EPA dust lead hazard levels. Out of 74 floor dust wipe samples, 10 XRF measurements were above the EPA standard of $10 \mu \mathrm{g} / \mathrm{ft}^{2}$ for floors as compared to 13 ICPMS analyzed samples (Table 3); that is, the XRF device produced 3 out of a possible 13 false negative results $(23.8 \%$ ) (Table 5). Out of 33 windowsill dust wipe samples, 6 XRF measurements were above the EPA standard of $100 \mu \mathrm{g} / \mathrm{ft}^{2}$ for windowsills, as compared to 9 ICPMS analyzed samples (Table 4); that is, the XRF device produced 3 out of a possible 9 false negative results $(33.3 \%)$ (Table 5). Considering ICPMS as the "gold standard," in total the XRF device produced 6 out of a possible 22 false negative results representing $27.3 \%$ (Table 5) and no false positive results.

A Bland Altman analysis was performed to formally test the comparability of XRF and ICPMS measurements for lead concentration in dust wipe samples. Prior to conducting the Bland-Altman analysis, data were tested for significant differences. Typically, this is done by testing the distribution of differences between methods. To determine whether this approach could be used with the dust data, the distribution of differences between ICPMS and XRF 
measurements were tested for normality (Table 6). Based on the parameters shown (Table 6) and the histogram (Figure 7), the distribution of differences between ICPMS and XRF measurements were not symmetric, not bell shaped and were highly skewed (skewness $=4.913)$. A ShapiroWilk normality test of the data further confirmed that the distribution of differences was nonnormal $(\mathrm{p}<0.001)$ (Table 7). Non-parametric tests were selected for subsequent analyses of lead concentration values determined by XRF and ICPMS.

To determine if there was a significant difference between the XRF and ICPMS measurements, a non-parametric Wilcoxon Signed Rank test was performed (Tables 8 and 9). Table 8 and 9 summarizes the results of the Wilcoxon Signed Rank test. In these analyses, XRF measurements did not differ significantly from ICPMS measurements $(\mathrm{Z}=-1.237, \mathrm{p}=0.216)$. The Bland Altman plot was constructed to determine the level of agreement.

To construct the plot (Figure 8), the acceptable limits of agreement were calculated using the mean of the difference and the standard deviation. The formula used was (SD*1.96) +Mean for the upper acceptable limit of agreement and Mean-(SD*1.96) for the lower acceptable limit of agreement. As shown in Figure 8, 4 of 107 points (3.74\%) were outside the acceptable limits of agreement.

From the Bland Altman plot, there is a cluster of scores where the mean is close to zero and the difference between the XRF and ICPMS is negative, which may indicate a proportional bias. Data from the Bland Altman analysis show that the bias between the XRF and ICPMS is $66.57 \mu \mathrm{g} / \mathrm{ft}^{2}$ which represents the mean of the difference. Thus, on average, the ICPMS measurements are $66.57 \mu \mathrm{g} / \mathrm{ft}^{2}$ greater than the XRF measurements. The positive bias appears to occur when measurements exceed $100 \mu \mathrm{g} / \mathrm{ft}^{2}$. For lower lead concentrations $\left(<100 \mu \mathrm{g} / \mathrm{ft}^{2}\right)$, the data are closer to each other. However, because the data used for Bland Altman analysis was 
non-normally distributed, the median of $0.37 \mu \mathrm{g} / \mathrm{ft}^{2}$ may be a better representation of the bias between the XRF and ICPMS. Also, a visual examination of the Bland Altman plot reveals a positive trend along the graph as shown in Figure 8, indicating that the difference between XRF and ICPMS results increases as the magnitude increases.

Exploratory analyses were conducted to generate hypothesis for future studies. An analysis was conducted to examine the difference in detected lead concentration in dust wipe samples collected from floors and windowsills. Lead concentrations determined by ICPMS were used for this analysis. As before, the data was assessed for normality. Based on the parameters shown (Table 10) and the histogram (Figure 9), the distribution of lead concentration in floor dust wipe samples were not symmetric, not bell shaped and was highly skewed (skewness = 5.576). A Shapiro-Wilk normality test of the data showed that the distribution of lead concentration in floor dust wipe samples was non-normal $(\mathrm{p}<0.001)$ (Table 11).

Based on the parameters shown (Table 12) and the histogram (Figure 10), the distribution of lead concentration in windowsill dust wipe samples were not symmetric, not bell shaped and were highly skewed (skewness $=4.224$ ). A Shapiro-Wilk normality test of the data showed that the distribution of lead concentration in windowsill dust wipe samples was non-normal $(\mathrm{p}<0.001)$ (Table 13).

The non-parametric Mann-Whitney U test was used to compare the differences between lead concentration in dust wipe samples collected from windowsills and from floors. Tables 14 and 15 summarizes the results of the Mann-Whitney U test. The mean rank was 43.93 for floor dust wipe samples and 76.59 for windowsill dust wipe samples (Table 14). The result of MannWhitney U test showed that lead concentration in dust wipe samples collected from windowsills 
were significantly higher than floor dust wipe samples as reported by the ICPMS device $\left(\mathrm{U}=475.50 \mu \mathrm{g} / \mathrm{ft}^{2}, \mathrm{p}<0.001\right)$ (Table 115). 
Table 1. Frequency distribution table of dust wipe samples collected and sample location

\begin{tabular}{|l|l|}
\hline Sample Location & Frequency \\
\hline Floor Samples & $74(69.2 \%)$ \\
\hline Windowsill Samples & $33(30.8 \%)$ \\
\hline Total & 107 \\
\hline
\end{tabular}

Table 2. Descriptive statistics for lead concentration in dust wipe samples measured by XRF and ICPMS, N=107

\begin{tabular}{|c|c|c|c|}
\hline & & XRF $\left(\mu g / f^{2}{ }^{2}\right)$ & ICPMS $\left(\mu \mathrm{g} / \mathbf{f t}^{2}\right)$ \\
\hline Mean & & 210.0940 & 276.6650 \\
\hline Std. Error o & ean & 90.0844 & 109.4991 \\
\hline Median & & 5.3000 & 2.3800 \\
\hline Std. Deviati & & 931.8399 & 1132.6672 \\
\hline Variance & & 868325.644 & 1282934.935 \\
\hline Skewness & & 5.383 & 5.068 \\
\hline Std. Error o & rewness & .234 & .234 \\
\hline Range & & 6083.35 & 7173.82 \\
\hline Minimum & & .00 & .03 \\
\hline Maximum & & 6083.35 & 7173.85 \\
\hline Percentiles & 25 & 4.9000 & .6700 \\
\hline & 50 & 5.3000 & 2.3800 \\
\hline & 75 & 10.1700 & 13.7100 \\
\hline
\end{tabular}


Table 3. Comparison of EPA-defined dust lead hazard concentration level detection in floor dust wipe samples

\begin{tabular}{|c|c|c|}
\hline & XRF & ICPMS \\
\hline Samples with Pb conc. $>\mathbf{1 0} \boldsymbol{\mu g} / \mathbf{f t}^{\mathbf{2}}$ & $10(13.5 \%)$ & $13(17.57 \%)$ \\
\hline Samples with Pb conc. $<\mathbf{1 0} \boldsymbol{\mu g} / \mathbf{f t}^{\mathbf{2}}$ & $64(86.49)$ & $61(82.43 \%)$ \\
\hline Total & 74 & 74 \\
\hline
\end{tabular}

Table 4. Comparison of EPA-defined dust lead hazard concentration level in windowsill dust wipe samples

\begin{tabular}{|c|c|c|}
\hline & XRF & ICPMS \\
\hline Samples with Pb conc. $>\mathbf{1 0 0} \boldsymbol{\mu g} / \mathbf{f t}^{\mathbf{2}}$ & $6(18.18 \%)$ & $9(27.27 \%)$ \\
\hline Samples with Pb conc. $<\mathbf{1 0 0} \boldsymbol{\mu g} / \mathbf{f t}^{\mathbf{2}}$ & $27(81.82 \%)$ & $24(72.73 \%)$ \\
\hline Total & 33 & 33 \\
\hline
\end{tabular}

Table 5. False Negative rates of XRF device

\begin{tabular}{|l|l|l|}
\hline Location & Frequency & Total \\
\hline Floor & $3(23.8 \%)$ & 13 \\
\hline Windowsill & $3(33.3 \%)$ & 9 \\
\hline Total & $6(27.3 \%)$ & 22 \\
\hline
\end{tabular}


Table 6. Descriptive statistics for the distribution of differences between ICPMS and XRF measurements, $\mathrm{N}=107$

\begin{tabular}{|c|c|c|c|c|}
\hline & & & Statistic & Std. Error \\
\hline ICPMS-XRF & Mean & & 66.5710 & 24.2484 \\
\hline & $95 \%$ Confidence & Lower Bound & 18.4963 & \\
\hline & Interval for Mean & Upper Bound & 114.6457 & \\
\hline & $5 \%$ Trimmed Mea & & 16.4380 & \\
\hline & Median & & .3700 & \\
\hline & Variance & & 62914.149 & \\
\hline & Std. Deviation & & 250.8269 & \\
\hline & Minimum & & -5.29 & \\
\hline & Maximum & & 1806.00 & \\
\hline & Range & & 1811.29 & \\
\hline & Interquartile Rang & & 8.73 & \\
\hline & Skewness & & 4.913 & .234 \\
\hline & Kurtosis & & 26.612 & .463 \\
\hline
\end{tabular}

Table 7. Summary statistics of Shapiro-Wilk normality test for difference between XRF and ICPMS measurements

\begin{tabular}{|l|c|c|c|}
\hline & \multicolumn{3}{|c|}{ Shapiro-Wilk } \\
\cline { 2 - 4 } & Statistic & df & Sig. \\
\hline ICPMS-XRF & .309 & 107 & .000 \\
\hline
\end{tabular}


Table 8. Summary of ranks for Wilcoxon signed rank test of XRF and ICPMS values

\begin{tabular}{|l|l|r|r|r|}
\hline \multicolumn{5}{|c|}{ Ranks } \\
\hline \multicolumn{2}{|c|}{} & \multicolumn{1}{|c|}{ N } & Mean Rank & Sum of Ranks \\
\hline ICPMS - XRF $\left(\mu \mathrm{g} / \mathrm{ft}^{2}\right)$ & Negative Ranks & $49^{\mathrm{a}}$ & 50.84 & 2491.00 \\
& Positive Ranks & $58^{\mathrm{b}}$ & 56.67 & 3287.00 \\
\hline & Ties & $0^{\mathrm{c}}$ & & \\
\hline & Total & 107 & & \\
\hline
\end{tabular}

a. ICPMS $\left(\mu \mathrm{g} / \mathrm{ft}^{2}\right)<\mathrm{XRF}$ highest average $\left(\mu \mathrm{g} / \mathrm{ft}^{2}\right)$

b. ICPMS $\left(\mu \mathrm{g} / \mathrm{ft}^{2}\right)>\mathrm{XRF}$ highest average $\left(\mu \mathrm{g} / \mathrm{ft}^{2}\right)$

c. $\operatorname{ICPMS}\left(\mu \mathrm{g} / \mathrm{ft}^{2}\right)=\mathrm{XRF}$ highest average $\left(\mu \mathrm{g} / \mathrm{ft}^{2}\right)$

Table 9. Results of Wilcoxon signed rank test comparing XRF and ICMPS values

\begin{tabular}{|l|c|}
\hline & ICPMS $\left(\boldsymbol{\mu g} / \mathbf{f t}^{\mathbf{2}}\right)-\mathbf{X R F}\left(\boldsymbol{\mu g} / \mathbf{f t}^{\mathbf{2}}\right)$ \\
\hline $\mathrm{Z}$ & $-1.237^{\mathrm{b}}$ \\
\hline Asymp. Sig. (2-tailed) & .216 \\
\hline
\end{tabular}

a. Wilcoxon Signed Ranks Test

b. Based on negative ranks. 
Table 10. Descriptive statistics for lead concentration in floor dust wipe samples measured by ICPMS, $\mathrm{N}=74$

\begin{tabular}{|c|c|c|c|c|}
\hline & & & Statistic & Std. Error \\
\hline ICPMS $\left(\mu \mathrm{g} / \mathrm{ft}^{2}\right)$ & Mean & & 226.8073 & 132.15239 \\
\hline & 95\% Confidence & Lower Bound & -36.5720 & \\
\hline & Interval for Mean & Upper Bound & 490.1866 & \\
\hline & $5 \%$ Trimmed Mea & & 9.2554 & \\
\hline & Median & & 1.2900 & \\
\hline & Variance & & 1292354.733 & \\
\hline & Std. Deviation & & 1136.81781 & \\
\hline & Minimum & & .03 & \\
\hline & Maximum & & 7173.85 & \\
\hline & Range & & 7173.82 & \\
\hline & Interquartile Rang & & 4.74 & \\
\hline & Skewness & & 5.576 & .279 \\
\hline & Kurtosis & & 30.969 & .552 \\
\hline
\end{tabular}

Table 11. Summary statistics of Shapiro-Wilk normality test for ICPMS measurements of floor dust wipe samples

\begin{tabular}{|l|r|r|r|}
\hline & \multicolumn{3}{|c|}{ Shapiro-Wilk } \\
\cline { 2 - 4 } & Statistic & df & \multicolumn{1}{|c|}{ Sig. } \\
\hline ICPMS $\left(\mu \mathrm{g} / \mathrm{ft}^{2}\right)$ & .200 & 74 & .000 \\
\hline
\end{tabular}


Table 12. Descriptive statistics for lead concentration in windowsill dust wipe samples measured by ICPMS, $\mathrm{N}=33$

\begin{tabular}{|c|c|c|c|c|}
\hline & & & Statistic & Std. Error \\
\hline \multirow[t]{13}{*}{ ICPMS $\left(\mu \mathrm{g} / \mathrm{ft}^{2}\right)$} & \multicolumn{2}{|l|}{ Mean } & 388.4673 & 197.16928 \\
\hline & \multirow{2}{*}{$\begin{array}{l}95 \% \text { Confidence } \\
\text { Interval for Mean }\end{array}$} & Lower Bound & -13.1534 & \\
\hline & & Upper Bound & 790.0880 & \\
\hline & \multicolumn{2}{|l|}{$5 \%$ Trimmed Mean } & 188.5856 & \\
\hline & \multicolumn{2}{|l|}{ Median } & 12.0300 & \\
\hline & \multicolumn{2}{|l|}{ Variance } & 1282898.984 & \\
\hline & \multicolumn{2}{|l|}{ Std. Deviation } & 1132.65131 & \\
\hline & \multicolumn{2}{|l|}{ Minimum } & .67 & \\
\hline & \multicolumn{2}{|l|}{ Maximum } & 6005.91 & \\
\hline & \multicolumn{2}{|l|}{ Range } & 6005.24 & \\
\hline & \multicolumn{2}{|l|}{ Interquartile Range } & 114.34 & \\
\hline & \multicolumn{2}{|l|}{ Skewness } & 4.224 & .409 \\
\hline & \multicolumn{2}{|l|}{ Kurtosis } & 19.795 & .798 \\
\hline
\end{tabular}

Table 13. Summary statistics of Shapiro-Wilk normality test for ICPMS measurements of windowsill dust wipe samples

\begin{tabular}{|l|r|r|r|}
\hline & \multicolumn{3}{|c|}{ Shapiro-Wilk } \\
\cline { 2 - 4 } & Statistic & df & \multicolumn{1}{|c|}{ Sig. } \\
\hline ICPMS $\left(\mu \mathrm{g} / \mathrm{ft}^{2}\right)$ & .382 & 33 & .000 \\
\hline
\end{tabular}


Table 14. Summary of ranks for Mann-Whitney U test for floor and windowsill lead concentrations

\begin{tabular}{|l|l|r|r|r|}
\hline \multicolumn{5}{|c|}{ Ranks } \\
\hline & Sample location & \multicolumn{1}{|c|}{ N } & Mean Rank & Sum of Ranks \\
\hline ICPMS $\left(\mu \mathrm{g} / \mathrm{ft}^{2}\right)$ & Floor & 74 & 43.93 & 3250.50 \\
\cline { 3 - 6 } & Window & 33 & 76.59 & 2527.50 \\
\cline { 2 - 5 } & Total & 107 & & \\
\cline { 2 - 5 } & & & & \\
\hline
\end{tabular}

Table 15. Results of Mann-Whitney U test comparing lead concentrations in floor and windowsill dust wipe samples

\begin{tabular}{|l|c|}
\hline \multicolumn{2}{|c|}{ Test Statistics $^{\mathbf{a}}$} \\
\hline & ICPMS $\left(\boldsymbol{\mu g} / \mathbf{f t}^{\mathbf{2}}\right)$ \\
\hline Mann-Whitney U & 475.500 \\
\hline Wilcoxon W & 3250.500 \\
\hline Z & -5.029 \\
\hline Asymp. Sig. (2-tailed) & .000 \\
\hline
\end{tabular}

a. Grouping Variable: Sample location 


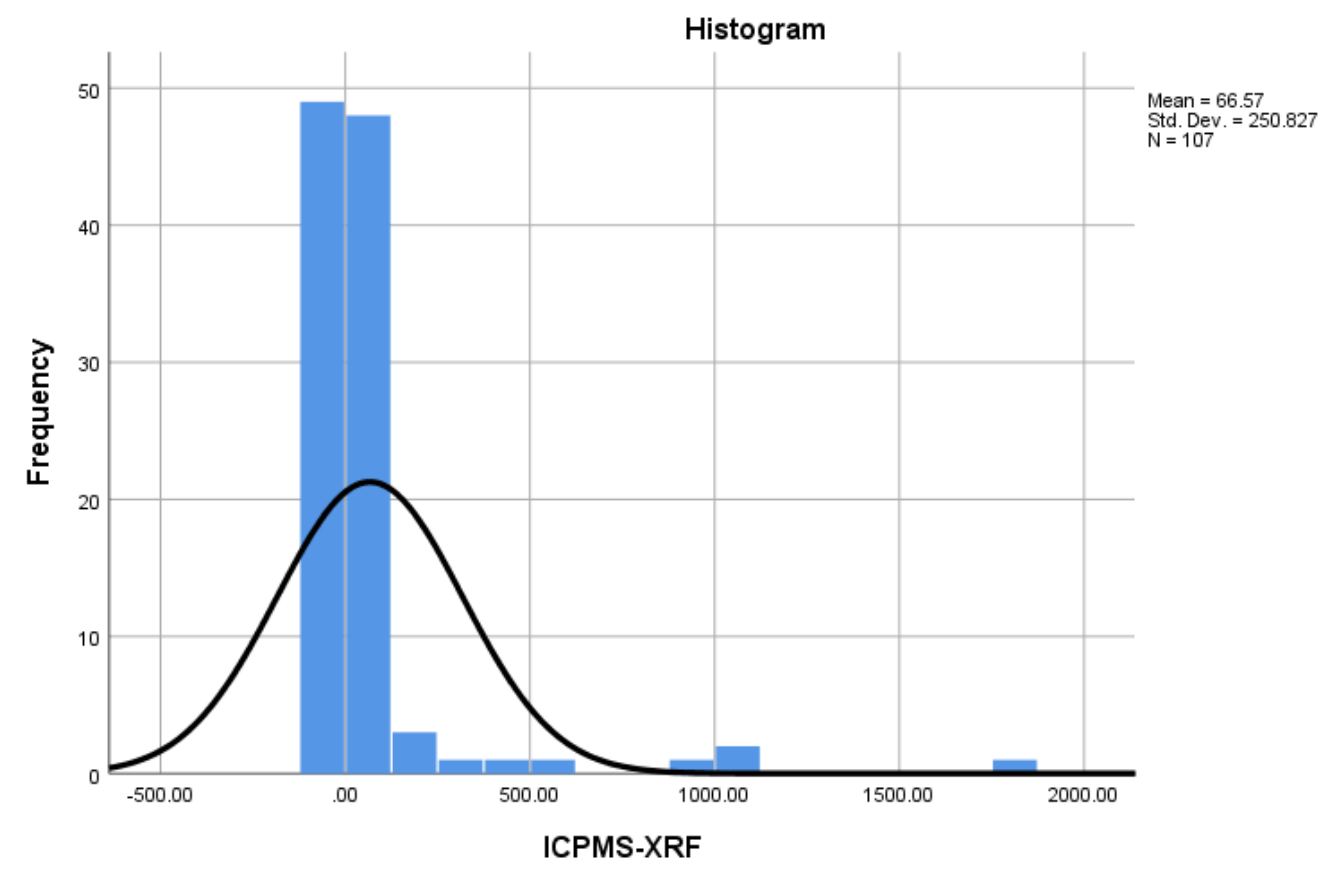

Figure 7. Distribution plot of differences between measurements by ICPMS and XRF with normality curve. 


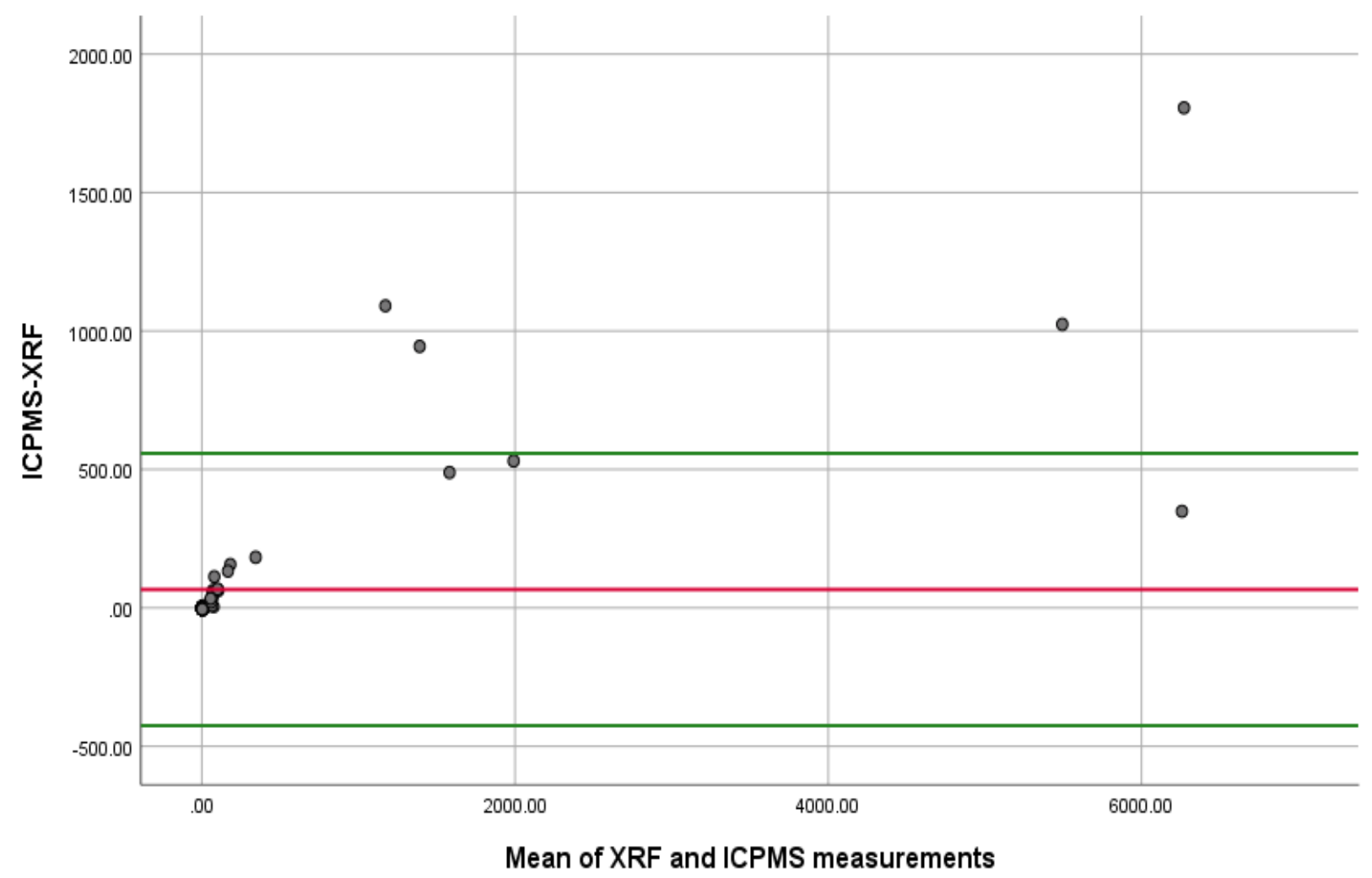

Figure 8. Bland-Altman plot showing the agreement between an XLp 300 XRF device and Agilent 7500cx ICPMS device for analyzing dust wipe samples for lead. 


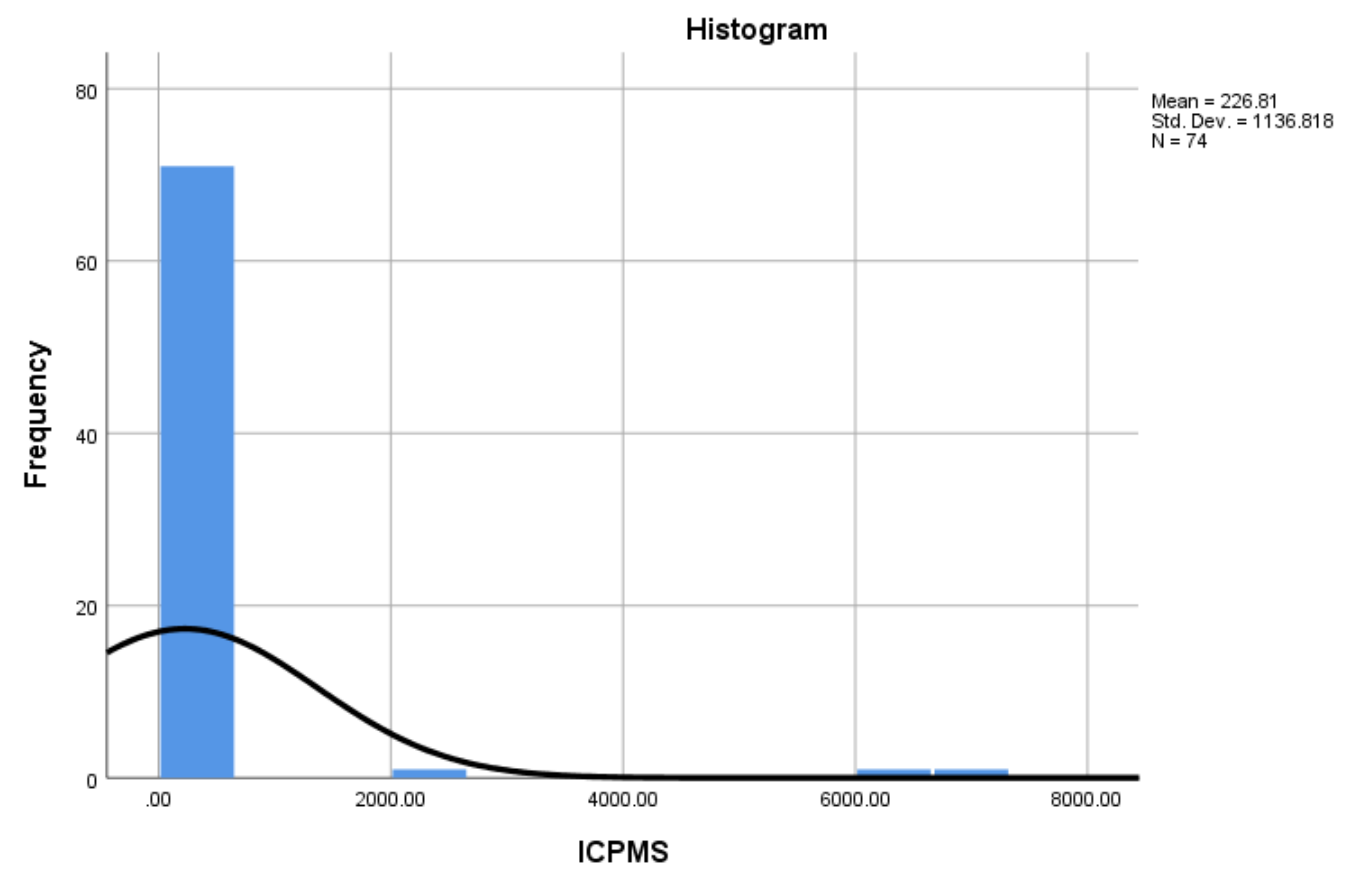

Figure 9. Distribution plot for ICPMS measurements of floor dust wipe samples with normality curve

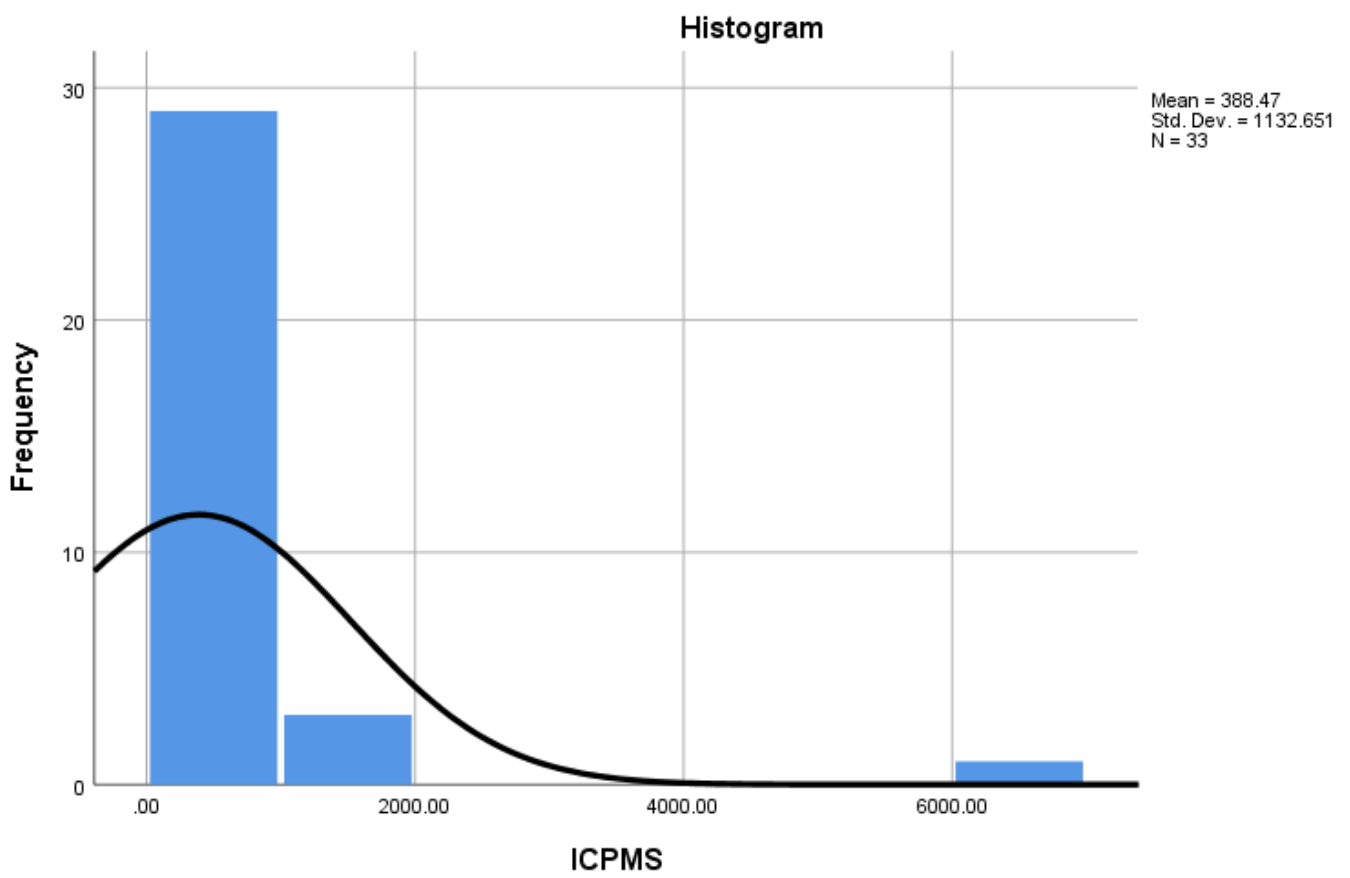

Figure 10. Distribution plot for ICPMS measurements of windowsill dust wipe samples with normality curve 


\section{CHAPTER 4 \\ DISCUSSION}

\subsection{Study Rationale}

The goal of this study was to validate the portable XRF device as a technique to measure lead concentrations in household dust wipe samples by testing the comparability of the XRF device and ICPMS.

In a previous study to validate the XRF device as an accurate technique to measure lead content in dust wipes (EPA, 2003), a comparison was performed between an XLt 700 XRF device and ICPAES. One-hundred and sixty dust wipe samples were analyzed with the XRF device and subsequently analyzed by ICPAES. In a similar study, Sterling et al. (2000), analyzed 185 dust wipe samples with an XRF device and then analyzed these same samples using FAAS.

In both studies, a good linear agreement (EPA, 2003: R=0.999; Sterling et al., 2000: $\mathrm{R}=0.93$ ) between the XRF and the laboratory method was used to conclude and establish the $\mathrm{XRF}$ as an accurate technique to measure lead content in dust wipes. However, a good correlation of results between two methods does not indicate agreement between the methods. Also, the comparison was against either the FAAS or ICPAES which are less precise. The lowest detection limits were $25 \mu \mathrm{g}$ and $20 \mu \mathrm{g}$ for FAAS and ICPAES respectively. More importantly, the experimental design of these studies was particularly focused on the former dust lead hazard standards set by the EPA which were $40 \mu \mathrm{g} / \mathrm{ft}^{2}$ for floors $250 \mu \mathrm{g} / \mathrm{ft}^{2}$ for windowsills.

For this study, the XRF device was compared to the currently most precise and accurate analytical method (ICPMS) for analyzing lead in field dust wipe samples, taking into consideration the new EPA dust lead hazard standards of $10 \mu \mathrm{g} / \mathrm{ft}^{2}$ for floors and $100 \mu \mathrm{g} / \mathrm{ft}^{2}$ for 
windowsills and window troughs (EPA, 2019). A Bland Altman analysis was selected to assess the agreement between the XRF device and the ICPMS. Determining the comparability of XRF measurements of dust wipe samples for lead and ICPMS analysis could eventually help inform future recommended methods for assessing critical child lead risks in the home environment.

\subsection{Summary of Major Findings}

From initial examination of the data, it was observed that the XRF underestimated the lead concentration in the dust wipe samples compared to the ICPMS analysis when the lead concentration was above $10 \mu \mathrm{g} / \mathrm{ft}^{2}$. At lead concentration levels below $10 \mu \mathrm{g} / \mathrm{ft}^{2}$, the XRF device generally gives a higher concentration than the ICPMS. This is similar to observations in the

studies by Sterling et al. (2000) and Harper et al. (2002). In the study by Sterling et al. (2000), the authors observed that XRF results are on the average $10 \%$ lower than FAAS measurements when lead concentrations in the dust wipe samples are above $300 \mu \mathrm{g}$. At the lower concentrations, the XRF results are $5 \%$ higher than FAAS concentrations.

The difference in measurements between the two methods at higher lead concentrations may be attributable to the multiple folding of the dust wipe which may cause an uneven distribution of lead through the wipe. The XRF measurements are performed on 4 quarters on the surface of the wipe (Figure 6). This means a low average lead concentration will be obtained if an abnormally low XRF reading is obtained from one quarter of the wipe due to the uneven distribution of dust in the wipe. Research has shown that the ability of the XRF device to analyze dust wipe samples is dependent on a uniform distribution of dust on the wipe material (Sterling et al., 2000). 
The Niton XLp 300 analyzer has a detection limit of $10 \mu \mathrm{g}$ (the lowest concentration of lead that can be reliably detected by the XRF analyzer) and as such any lead concentration detected below $10 \mu \mathrm{g}$ is merely an estimate and not reliable. This may be an explanation as to why, at lead concentration levels below $10 \mu \mathrm{g} / \mathrm{ft}^{2}$, the XRF device appears to give a higher concentration than the ICPMS. More research is required to confirm and explain this difference between XRF device and laboratory measurements.

As the calls for further action from the EPA to lower the new dust lead hazard standard from $10 \mu \mathrm{g} / \mathrm{ft}^{2}$ to $5 \mu \mathrm{g} / \mathrm{ft}^{2}$ for floors increase (American Academy of pediatrics, 2016), there is the need to manufacture XRF devices with detection limits lower than $5 \mu \mathrm{g}$ to accurately capture lead contamination in household dust wipe samples.

\subsubsection{Comparison of EPA-defined dust lead hazard concentration level detection in dust wipe samples}

An important part of method comparison is to understand the false positive and false negative rates of the measurement system. The XRF was evaluated using the new EPA dust lead hazard standards of $10 \mu \mathrm{g} / \mathrm{ft}^{2}$ for floors and $100 \mu \mathrm{g} / \mathrm{ft}^{2}$ for windowsills (EPA, 2019). For this study, a false negative result was defined as an XRF lead concentration reading below the standard levels of $10 \mu \mathrm{g} / \mathrm{ft}^{2}$ and $100 \mu \mathrm{g} / \mathrm{ft}^{2}$, when the true concentration was higher. A false positive result was defined as an XRF lead concentration reading above the standard levels of $10 \mu \mathrm{g} / \mathrm{ft}^{2}$ and $100 \mu \mathrm{g} / \mathrm{ft}^{2}$ when the true concentration was lower. As shown in Table 5 , the XRF produced a false negative ratio of $23.8 \%$ for floor dust wipe samples and $33.3 \%$ for windowsill dust wipe samples. Thus, the mean false negative rate of $27.3 \%$ was produced by the XRF device. Interestingly, there were no false positive results from the XRF device. These rates are both less than the false positive and false negative rates presented in other studies. For example, 
in the study by the US EPA (2003), the XRF device produced $35.3 \%$ false negative results and $18.4 \%$ false positive results. The authors concluded that the XRF device is slightly negatively biased (EPA, 2003), a conclusion which may be made for the results presented in this study. It is important to note that, in determining the false positive and false negative rates for the EPA (2003) study, the former dust lead hazard standards of $40 \mu \mathrm{g} / \mathrm{ft}^{2}$ for floors $250 \mu \mathrm{g} / \mathrm{ft}^{2}$ for windowsills were used. Additional studies designed with regard to detecting lead concentrations in dust wipe samples at the new dust lead hazard standards, are needed to make a better comparison of false positive and false negative rates of the XRF device. These studies should have majority of samples at or near the new EPA dust lead hazard standards of $10 \mu \mathrm{g} / \mathrm{ft}^{2}$ and $100 \mu \mathrm{g} / \mathrm{ft}^{2}$ for floors and windowsills respectively.

\subsubsection{Bland Altman analysis of XRF and ICPMS measurements of lead concentration in dust wipe samples}

A thorough review of literature revealed no currently available peer reviewed studies assessing the comparability of the XRF to the ICPMS device for analyzing dust wipe samples. This study was conducted to fill that gap and assess comparability of XRF and ICPMS using the Bland Altman approach. Bland and Altman (1986) recommended that $95 \%$ of data points on the Bland Altman plot must fall within the established lower and upper limits of agreement, or $\pm 2 \mathrm{SD} s$ of the mean difference, to establish an acceptable agreement between the two methods. From the plot, 103 out of 107 representing $96.3 \%$ of the data points were within the lower and upper limits of agreement. The results of the study indicated that the XLp 300 XRF device has a

good agreement with the Agilent 7500cx ICPMS device within the lead concentrations measured however a positive bias existed when the lead concentration exceed $100 \mu \mathrm{g} / \mathrm{ft}^{2}$. For lead concentrations below $100 \mu \mathrm{g} / \mathrm{ft}^{2}$ a negative bias existed between the XRF and ICPMS. 


\subsection{Comparison of lead concentration in floor and windowsill samples (Exploratory}

analyses)

This study was not designed to examine the difference in detected lead concentrations between floor and windowsill dust wipe samples, so the data collected may not provide conclusive results for this type of analysis. However, the exploratory analysis to compare lead concentrations in floor and windowsill samples was conducted to generate hypothesis for future studies.

Results from the exploratory analyses indicated that windows may be a higher lead exposure risk than floors. The Mann-Whitney U test to compare the lead concentrations in dust wipe samples collected from windows and floors revealed that dust from windows had significantly higher lead concentrations than dust on floors of the participating homes.

The reason for this difference in lead concentration could be from the fact that windows could potentially collect more settled dust than floors. Windows, when open may collect dust from exterior sources that may be contaminated with lead as well as concentrations within the home. Millions of homes in the US, especially in low-income areas, have old windows which usually have lead paint (Cox et al. 2011). Many of the homes sampled for this study were built before 1978, were in low income areas, and had old windows. Constantly opening and closing these windows causes friction which generates lead contaminated dust that may settle on the windows. Additionally, floor dust samples, similar to many of the floor samples collected for this study, were from open areas in the living rooms, kitchens, bathrooms, and bedrooms of the participating homes. These areas are subject to human traffic which may disperse or transfer the 
lead contaminated dust to other less accessible areas of rooms and may be missed in sampling. Additional studies involving whole room dust sampling with vacuums may provide better understanding of this issue and could validate this claim.

\subsection{Implications of study findings for public health}

The findings of this study have several implications for public health. The finding that the XRF has a good agreement with ICPMS indicates that the XRF is reporting a correct value of lead concentration at lower lead concentration levels. If confirmed by replicate studies, the XRF could serve as a cost-effective device for dust wipe analysis that provides timely results. This is particularly important since interior dust lead loadings have been shown to be reliable predictors of elevated blood lead levels in children (Davies et al., 1990; Lanphear et al., 1995, 1998;

Gaitens et al., 2009). The ability of the XRF to produce rapid, readily available, relatively lowcost results regarding interior dust lead loadings, is critical for the detection of child lead hazards in the home.

The findings are also important for lead hazard clearance testing. Lead hazard clearance testing refers to the requirement that a certified Lead Risk Assessor verifies that previous elevated levels of lead in dust have been reduced enough to make the residence safe for dwellers, typically at the conclusion of lead hazard control interventions (US EPA, 2001). The risk assessor may not need to send the dust wipe samples for laboratory analysis at lower levels of lead concentration, which would reduce the time and costs associated with clearing a home as "safe" for residents.

Results from the exploratory analysis conducted showed that dust from windows have significantly more lead than dust from floors, raises further concern about the EPA dust lead 
hazard standards especially for windowsills. The new EPA dust lead hazard standards $\left(10 \mu \mathrm{g} / \mathrm{ft}^{2}\right.$ for floors; $100 \mu \mathrm{g} / \mathrm{ft}^{2}$ for windowsills) were set to prioritize reducing floor dust lead loadings over windowsill dust lead loadings. Floors take up more square footage of a house and children spend most of their time on floors rather than windowsills and hence have a bigger impact on children's exposure to lead. The theory that dust from floors poses more risk to children than dust from windowsills formed the primary basis for the decision by the EPA in setting the new dust lead hazard standards.

However, this may not be the case for several homes across the US. For example, in some of the participating homes in this study, children slept in beds that were at the height of their windowsills and positioned next to them. These windows were easily accessible to the children and some of the children had put their toys and other items on the windowsills. This was found to be a common arrangement in homes we visited in El Paso and may be common in many other cities with neighborhoods at high risk of lead exposure. These windows may be a lot more accessible to children than predicted by the EPA, and the children in these homes may be more exposed to lead than the EPA projects.

These findings, in addition to toxicological studies showing that the current dust lead hazard standards are able to produce BLLs higher than $5 \mu \mathrm{g} / \mathrm{dl}$ in children (Gilbert \& Weiss, 2006), strengthens the call by the American Academy of Pediatrics on the EPA to further lower the dust lead hazard standards for windows to $50 \mu \mathrm{g} / \mathrm{ft}^{2}$.

The exploratory results suggested that dust from windows may have significantly more lead concentration than dust from floors. This finding merits a review of the current educational strategy on preventing lead exposure in children. An important recommendation for reducing lead exposure in the home is "wet mopping floors, wet wiping windows, and surfaces every 
week to control dust." Some of the participating homes in this study had old windows and lots of visibly settled dust. Modifying the educational strategy to stress cleaning windows frequently, particularly when seeing settled dust on older windows, could further reduce the likelihood that children in these homes are exposed to lead.

Overall, the results of the study may have broader implications for public health, however additional studies on XRF comparability with ICPMS at higher lead levels and contribution of floor and windowsill dust lead loadings to child blood levels are needed.

\subsection{Limitations of the study}

There are a few limitations of this study that should be considered. Using field dust wipe samples has some disadvantages such as a greater inherent variability which is evident in this study. Also, field dust wipe samples may have extraneous materials such as paint chips and organic matter. Some samples collected from these homes had human hair and other materials. For future studies, developing methods for laboratory generated samples may help to avoid this limitation.

Over $70 \%$ of the dust wipe samples had lead concentration below $10 \mu \mathrm{g}$ and less than $30 \%$ had a significant lead concentration $(>10 \mu \mathrm{g})$. Thus, these data may not adequately represent data relevant to the new EPA dust lead hazard standards of $10 \mu \mathrm{g} / \mathrm{ft}^{2}$ for floors and $100 \mu \mathrm{g} / \mathrm{ft}^{2}$. Additional studies using several samples with lead concentrations close to the new dust lead hazard standards $(10 \mu \mathrm{g} / \mathrm{ft} 2$ for floors; $100 \mu \mathrm{g} / \mathrm{ft} 2$ for windowsills) could avoid this limitation. Again, laboratory generated samples with known lead concentrations may also be a solution.

Also, the XLp 300 XRF device used in this study had a lowest detection limit of $10 \mu \mathrm{g}$. This means over $70 \%$ of the lead concentrations in the dust wipe samples reported by the XRF 
are estimates and may not be reliable. Using an XRF device with a detection limit less than $10 \mu \mathrm{g}$ may avoid this limitation. 


\section{CONCLUSION}

In-depth studies on comparison of lead concentration in dust from windowsills and floors would be very helpful. Further research must adopt an appropriate sample collection protocol, analysis plan, and hypothesis set around comparing lead concentrations in floor and windowsill dust wipe samples.

The comparability of the X-ray fluorescence device and inductively coupled plasma mass spectrometry reported in this study suggest that the XRF device is appropriate for measuring lead concentrations in field dust wipe samples for lower levels of household lead concentrations. It can also be concluded that XRF device may be used as a positive and negative screen for lead dust hazards in the homes of children. This is particularly important since interior lead dust loadings have been shown to be reliable predictors of elevated blood lead levels in children. Replicate studies are required to confirm these conclusions. If confirmed, the XRF device may be a reliable measure for clearance evaluation and lead risk assessment at the new EPA dust lead hazard standards. 


\section{MPH PROGRAM FOUNDATIONAL COMPETENCIES}

BIOSTATISTICS. Biostatistics is the development and application of statistical reasoning and methods in addressing, analyzing and solving problems in public health; health care; and biomedical, clinical and population-based research.

1. Apply descriptive techniques commonly used to summarize public health data.

2. Apply common statistical methods for inference.

3. Develop written and oral presentations based on statistical analyses for both public health professionals and educated lay audiences.

ENVIRONMENTAL HEALTH SCIENCES CORE COMPETENCIES. Environmental health sciences represent the study of environmental factors including biological, physical and chemical factors that affect the health of a community. Upon graduation, the MPH student should be able to........ 1. Describe the direct and indirect human, ecological and safety effects of major environmental and occupational agents.

2. Describe federal and state regulatory programs, guidelines and authorities that control environmental health issues.

3. Specify current environmental risk assessment methods.

4. Specify approaches for assessing, preventing and controlling environmental hazards that pose risks to human health and safety.

\section{HISPANIC/BORDER HEALTH CONCENTRATION COMPETENCIES}

1. State and discuss the current major communicable, non-communicable, and environmental public health threats in Hispanic and border communities

\section{MPH FOUNDATIONAL COMPETENCIES}

Evidence-based Approaches to Public Health

1. Interpret results of data analysis for public health research, policy or practice 


\section{REFERENCES}

Adams M. J., Ewen G. J., and Shand C. A. (1988) "Acquisition and analysis of GFAAS data," Journal of Automatic Chemistry, vol. 10, no. 3, pp. 130-134. https://doi.org/10.1155/S1463924688000240.

Allabashi, W. Stach, A. de la Escosura-Muñiz, L. Liste-Calleja, A. Merkoçi (2009) ICP-MS: a powerful technique for quantitative determination of gold nanoparticles without previous dissolving J. Nanopart. Res., 11, pp. 2003-2011

American Academy of Pediatrics. 2016. Prevention of Childhood Lead Toxicity. Council on Environmental Health. Pediatrics Jul;138(1). [accessed 2019 Oct. 13]. Available from: http://pediatrics.aappublications.org/content/early/2016/06/16/peds.2016-1493.

American Society for Testing and Materials, West Conshohocken, PA (1998). A Guide for Evaluating the Performance Characteristics of Qualitative Chemical Spot Test Kits for Lead in Paint, ASTM Standard E 1828, Annual Book of Standards, Vol. 04.07, West Conshohocken, PA

ASTM D7659-10 (2015) Standard Guide for Strategies for Surface Sampling of Metals and Metalloids for Worker Protection, ASTM International, West Conshohocken, PA, 2015, https://doi.org/10.1520/D7659-10R15

ASTM Standard E1792-96a (2015): Standard Specification for Wipe Sampling Materials for Lead in Surface Dust, Annual Book of ASTM Standards, Vol. 04.11, ASTM International, West Conshohocken, PA, pp. 1162-1164.

Badiei, H. R., \& Karanassios, V. (2000). Rhenium-cup in-torch vaporization inductively coupled plasma atomic emission spectrometry for liquid, slurry or solid micro-samples. Journal of Analytical Atomic Spectrometry, 15(9), 1057-1062. doi:10.1039/B001781G

Beauchemin D, (2017). Encyclopedia of Spectroscopy and Spectrometry (Third Edition), pg 236-245https://doi.org/10.1016/B978-0-12-409547-2.11222-3

Bland, J.M., Altman (1986), D.G. Statistical methods for assessing agreement between two methods of clinical measurement. Lancet, 1986. i: p. 307-310.

Borges, D. L.G., A. F. Da Sila, B. Welz, A. J. Curtius, and U. Heitmann (2006). Determination of lead in biological samples by high-resolution continuum source graphite furnace atomic absorption spectrometry with direct solid sampling. Journal of Anal. Atom. Spectrom. 21: 763-769. [Crossref], [Web of Science ®], [Google Scholar]

Butcher, D.J., and Sneddon, J. (1998) A practical guide to graphite furnace atomic absorption spectrometry. Wiley, New York

Butcher D.J., (2017) Recent highlights in graphite furnace atomic absorption spectrometry, Applied Spectroscopy Reviews, 52:9, 755-773, DOI: 10.1080/05704928.2017.1303504 
Caldwell K.L., Cheng P.Y., Jarrett J.M., Makhmudov, A., Vance, K., Ward, C.D., Jones, R.L., Mortensen, M.E.. (2017) Measurement Challenges at Low Blood Lead Levels. Pediatrics. 140(2):e20170272. https://www.ncbi.nlm.nih.gov/pubmed/28771411

Carey, J. M., \& Caruso, J. A. (1992). Electrothermal vaporization for sample introduction in plasma source spectrometry. Critical Reviews in Analytical Chemistry, 23(5), 397-439. doi:10.1080/10408349208051652

CDC (1991). Preventing lead poisoning in young children. Atlanta, GA: US Department of Health and Human Services.

CDC (1997). Children with elevated blood lead levels attributed to home renovation and remodeling activities-New York, 1993-1994. MMWR Morb Mortal Wkly Rep. 45:1120-3. https://www.cdc.gov/nceh/lead/data/learnmore.htm

CDC (2012). Advisory Committee on Childhood Lead Poisoning Prevention. low level lead exposure harms children: a renewed call for primary prevention. Atlanta, GA: Centers for Disease Control and Prevention; Available at: http://www.cdc.gov/nceh/lead/ACCLPP/Final_Document_030712.pdf.

CDC (2012). Healthy Homes and Lead Poisoning Prevention: what do parents need to know to protect their children? Atlanta, GA: US Department of Health and Human Services. https://www.cdc.gov/nceh/lead/acclpp/blood_lead_levels.htm

CDC, (2019) Centers for Disease Control and Prevention Childhood Lead Poisoning Prevention Program (2019). https://www.cdc.gov/nceh/lead/default.htm

Charney E, Sayre J, Coulter M (1980). Increased lead absorption in inner city children: where does the lead come from?. Pediatrics.;65(2):226-231

Cheng-Mau Wu, Hung-Teh Tsai, Kai-Hsing Yang \& Jet-Chau Wen (2012): How Reliable is XRay Fluorescence (XRF) Measurement for Different Metals in Soil Contamination?, Environmental Forensics, 13:2, 110-121. http://dx.doi.org/10.1080/15275922.2012.676603

Clark, S., Menrath, W., Chen, M., Roda, S., \& Succop, P. (1999). Use of a field portable X-ray fluorescence analyzer to determine the concentration of lead and other metals in soil samples, Ann Agric Environ Med, 6, 27-32.

Consumer Product Safety Commission (CSPC) (2015), Study on the Applicability of X-ray Fluorescence Spectrometry for Use in ASTM F963 Total Element Screening. https://www.cpsc.gov/s3fs-public/pdfs/Lead-in-Paint-Measure-April2015.pdf (Accessed Nov 25 2019)

Cornwell DA, Brown RA, Via SH. (2016). National survey of lead service line occurrence. J. Am. Water Works Assoc. 108:E182-91

Cox C, Dewalt G, O’Haver R, Salatino B. (2011). American Healthy Homes Survey Lead and Arsenic Findings. Washington, DC: US Dep. Hous. Urban Dev. 
Dewalt F.G., Cox D.C., O’Haver R., Salatino B., Holmes D., Ashley P.J., Pinzer E.A., Friedman W., Marker D., Viet S.M., Fraser A. (2015). Prevalence of Lead Hazards and Soil Arsenic in U.S. Housing. Journal of Environmental Health. 78(5): 22-29. http://www.neha.org/node/6429

Dignam, T., Pomales, A., Werner, L., Newbern, E. C., Hodge, J., Nielsen, J., . . Brown, M. J. (2018). Assessment of child lead exposure in a philadelphia community, 2014. Journal of Public Health Management and Practice: JPHMP, doi:10.1097/PHH.0000000000000711

Dignam, T., Kaufmann, R. B., LeStourgeon, L., \& Brown, M. J. (2019). Control of Lead Sources in the United States, 1970-2017: Public Health Progress and Current Challenges to Eliminating Lead Exposure. Journal of public health management and practice : JPHMP, 25 Suppl 1, Lead Poisoning Prevention(Suppl 1 LEAD POISONING PREVENTION), S13-S22. doi:10.1097/PHH.0000000000000889

Eckel, W. P., Rabinowitz, M. B., \& Foster, G. D. (2001). Discovering unrecognized leadsmelting sites by historical methods. American journal of public health, 91(4), 625-627. doi:10.2105/ajph.91.4.625

Elliott J.R, Frickel S. (2013). The historical nature of cities: a study of urbanization and hazardous waste site accumulation. Am. Sociol. Rev. 78:521-43

EPA (1994). "Method 200.8: Determination of Trace Elements in Waters and Wastes by Inductively Coupled Plasma-Mass Spectrometry," Revision 5.4. Cincinnati, OH

EPA (1995) A Field Test of Lead-Based Paint Testing Technologies: Technical Report, EPA 747-R-95-002b.

EPA (2001) Lead; Identification of Dangerous Levels of Lead; Final Rule. Federal Register (66 FR 1206, January 5, 2001) (FRL-6763-5)

EPA (2002). Environmental Technology Verification Report: Lead in Dust Wipe Measurement Technology, Niton Corporation X-Ray Fluorescence Spectrum Analyzer XL-300 Series. https://nepis.epa.gov/Exe/ZyPDF.cgi/P1001017.PDF?Dockey=P1001O17.PDF

EPA (2003). Environmental Technology Verification Report: Lead in Dust Wipe Measurement Technology, Niton LLC X-Ray Fluorescence Spectrum Analyzer XLt-700 Series. https://archive.epa.gov/nrmrl/archive etv/web/pdf/01_vr_niton_700.pdf

EPA (2007), Method 6200: Field Portable X-Ray Fluorescence Spectrometry for The Determination of Elemental Concentrations in Soil and Sediment. https://www.epa.gov/sites/production/files/2015-12/documents/6200.pdf (Accessed Dec 02, 2019)

EPA (2008) Lead: Renovation, Repair and Painting (LRRP) Rule

EPA (2019) Review of the dust-lead hazard standards and the definition of Lead-Based paint. (84 FR 32632, July 9, 2020) (FRL-9995-49) 
Gaitens, J. M., Dixon, S. L., Jacobs, D. E., Nagaraja, J., Strauss, W., Wilson, J. W., \& Ashley, P. J. (2009). Exposure of U.S. children to residential dust lead, 1999--2004: I. housing and demographic factors. Environmental Health Perspectives, 117(3), 461-467. Retrieved from http://0 search.ebscohost.com.lib.utep.edu/login.aspx?direct=true \&db=a9h\&AN=37254178\&site $=$ ehost-live \&scope $=$ site

Gilbert, S. G., \& Weiss, B. (2006). A rationale for lowering the blood lead action level from 10 to $2 \mathrm{microg} / \mathrm{dL}$. Neurotoxicology, 27(5), 693-701. doi:10.1016/j.neuro.2006.06.008

Gulson, B., \& Taylor, A. (2017). A simple lead dust fall method predicts children's blood lead level: New evidence from Australia doi:https://doi.org/10.1016/j.envres.2017.07.047

Harper, M., Hallmark, T. S., \& Bartolucci, A. A. (2002). A comparison of methods and materials for the analysis of leaded wipes. Journal of Environmental Monitoring, 4(6), 1025-1033. doi:10.1039/B208456M

Health Impact Proj. (2017). 10 policies to prevent and respond to childhood lead exposure: an assessment of the risks communities face and key federal, state, and local solutions. Rep., Pew Charit. Trusts, Philadelphia, PA

Hybrivet (2006) LeadCheck Swabs. Available http://www.leadcheck.com/

IUPAC (1997). Compendium of Chemical Terminology, 2nd ed. (the "Gold Book"). Compiled by A. D. McNaught and A. Wilkinson. Blackwell Scientific Publications, Oxford Online version (2019-) created by S. J. Chalk. ISBN 0-9678550-9-8. https://doi.org/10.1351/goldbook

Korfmacher, K. S., \& Dixon, S. (2007). Reliability of spot test kits for detecting lead in household dust. Environmental Research, 104(2), 241-249. Retrieved from http://0search.ebscohost.com.lib.utep.edu/login.aspx?direct=true \&db=cmedm\&AN=1743 $\underline{4162 \& \text { site }=\text { ehost-live \&scope }=\text { site }}$

Lanphear, B. P., Emond, M., Jacobs, D. E., Weitzman, M., Tanner, M., Winter, N. L., . . . Eberly, S. (1995). A side-by-side comparison of dust collection methods for sampling lead-contaminated house dust doi:https://doi.org/10.1006/enrs.1995.1015

Lanphear, B. P., \& Roghmann, K. J. (1997). Pathways of lead exposure in urban children doi: https://doi.org/10.1006/enrs.1997.3726

Lanphear, B. P., Matte, T. D., Rogers, J., Clickner, R. P., Dietz, B., Bornschein, R. L., . . . Jacobs, D. E. (1998). The contribution of lead-contaminated house dust and residential soil to children's blood lead levels: A pooled analysis of 12 epidemiologic studies doi: https://doi.org/10.1006/enrs.1998.3859

Lanphear, B. P., Rauch, S., Auinger, P., Allen, R. W., \& Hornung, R. W. (2018). Low-level lead exposure and mortality in US adults: A population-based cohort study doi: https://doi.org/10.1016/S2468-2667(18)30025- 
Lemaire, R., Bianco, D. D., Garnier, L., \& Beltramo, J. L. (2013). Determination of lead in lipstick by direct solid sampling high-resolution continuum source graphite furnace atomic absorption spectrometry: Comparison of two digestion methods. Analytical Letters, 46(14), 2265-2278. doi:10.1080/00032719.2013.787430

Markey, A., Clark, C., Succop, P., \& Roda, S. (2008). Determination of the Feasibility of Using a Portable X-Ray Fluorescence (XRF) Analyzer in the Field for Measurement of Lead Content of Sieved Soil. Journal of Environmental Health, 70(7), 24-30. Retrieved from www.jstor.org/stable/26327571

Markowitz G, Rosner D. (2013). Lead Wars: The Politics of Science and the Fate of America's Children. Berkeley: Univ. Calif. Press

Muller, C., Sampson, R. J., \& Winter, A. S. (2018). Environmental inequality: The social causes and consequences of lead exposure. Annual Review of Sociology, 44(1), 263-282. doi:10.1146/annurev-soc-073117-041222

Muller, Y., Favreau, P., \& Kohler, M. (2014). Performance evaluation of currently used portable $\mathrm{X}$ ray fluorescence instruments for measuring the lead content of paint in field samples. Journal of Occupational and Environmental Hygiene, 11(8), 528-537. doi:10.1080/15459624.2014.880788

National Institute for Occupational Safety and Health (NIOSH). (1998) Lead by field portable XRF: Method 7702. NIOSH/DSHEFS, NIOSH Manual of Analytical Methods (NMAM), fourth ed., 1:1-4. Available at: http://www.cdc.gov/niosh/nmam/pdfs/7702.pdf

National Institute of Standards (NIST), Rossiter WJ, Vangel MG, McKnight ME, Dewalt G. (2000) Spot Test Kits for detecting lead in household paint: A laboratory evaluation National Institute of Standards and Technology. U. S. Department of Commerce; Washington, DC:. NISTIR 6398

Needleman HL (2000). The removal of lead from gasoline: historical and personal reflections. Environ Res.;84:20-35.

Nelms M. S. (2005). Inductively Coupled Plasma Mass Spectrometry Handbook. Blackwell Publishing Ltd. |DOI:10.1002/9781444305463

Niton, “Analyzing Thin Samples," XL-309 \& 700 Series User’s Guide Version 5.0, ThermoFisher Scientific.

Pell M. B., Schneyer J. (2016). Off the charts: the thousands of U.S. locales where lead poisoning is worse than in Flint. Reuters, Dec. 19. http://www.reuters.com/investigates/special-report/usa-lead-testing/

Raymond, J., \& Brown, M. J. (2017). Childhood blood lead levels in children aged $<5$ years -united states, 2009-2014. MMWR Surveillance Summaries, 66(3), 1-7. Retrieved from http://0-

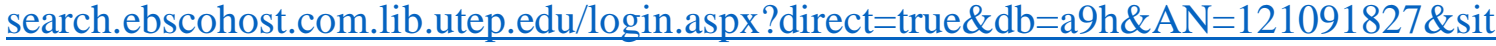
e=ehost-live\&scope $=$ site 
Reames, G., \& Lance, L. L. (2002). Childhood lead poisoning investigations: Evaluating a portable instrument for testing soil lead. Journal of Environmental Health, 64(8), 9. Retrieved from http://0search.ebscohost.com.lib.utep.edu/login.aspx?direct=true $\& d b=c m e d m \& A N=11930816 \&$ site=ehost-live $\&$ scope $=$ site

Reece R. M., Reed A.J., Clark C.S., Angoff R.C., Casey K.R., Challop R.S., McCabe E. (1972): Elevated blood lead and the in situ analysis of wall paint by x-ray fluorescence. Am J Dis Children, 24, 500-502.

Resano M, Garcia-Ruiz E. (2011) High-resolution continuum source graphite furnace atomic absorption spectrometry: Is it as it sounds? A critical review. Anal Bioanal Chem.;399:323-330. [PubMed] [Google Scholar]

Rogers, J., Viet, S., Roda, S., Fraser, A., Friedman, W., Zhou, J., and Jacobs, D. (2012). Statistical Comparison of Analysis Results for Lead on Dust Wipe Samples by X-ray Fluorescence Analysis and Flame Atomic Absorption Spectrometry. Journal of ASTM International, 9 (5), 1-12. Retrieved from https://doi.org/10.1520/JAI104196

Safruk, A. M., McGregor, E., Whitfield Aslund, M. L., Cheung, P. H., Pinsent, C., Jackson, B. J., ... Sigal, E. A. (2017). The influence of lead content in drinking water, household dust, soil, and paint on blood lead levels of children in flin flon, manitoba and creighton, saskatchewan. Science of the Total Environment, 593/594, 202-210. doi:10.1016/j.scitotenv.2017.03.141

Schatzlein D, Thomsen V, Mercuro D., (2003) Theory and Use of Field Portable X-Ray Fluorescence for Soil Analvsis workshop.

Silva, A. F., D. L. G. Borges, F. G. Lepri , B. Welz, A. J. Curtius, and U. Heitamnn . (2005). Determination of cadmium in coal using solid sampling graphite furnace high-resolution continuum source atomic absorption spectrometry, Anal. Bioanal. Chem. 382: 1835 1841. [Crossref], [PubMed], [Web of Science $®]$, [Google Scholar]

Skröder, H., Hawkesworth, S., Moore, S. E., Wagatsuma, Y., Kippler, M., \& Vahter, M. (2016). Prenatal lead exposure and childhood blood pressure and kidney function. Environmental Research, 151, 628-634. doi:10.1016/j.envres.2016.08.028

Smith RC, Daley MR. (1987) Domestic Secondary Lead Industry: Production and Regulatory Compliance Costs. Washington, DC: Bureau of Mines, US Dept of Interior; 1987. Information Circular 9156.

Sobin, C., Flores-Montoya, M. G., Gutierrez, M., Parisi, N., \& Schaub, T. (2015). $\delta$ Aminolevulinic acid dehydratase single nucleotide polymorphism 2 (ALAD2) and peptide transporter $2 * 2$ haplotype (hPEPT $2 * 2$ ) differently influence neurobehavior in low-level lead exposed children. Neurotoxicology and Teratology, 47, 137-145. http://doi.org/10.1016/j.ntt.2014.12.001 
Sobin, C., Montoya, M. G. F., Parisi, N., Schaub, T., Cervantes, M., \& Armijos, R. X. (2013). Microglial disruption in young mice with early chronic lead exposure. Toxicology Letters, 220(1), 44-52. http://doi.org/10.1016/j.toxlet.2013.04.003

Standard Metal Directory (1931, 1940, 1946, 1950, 1954, 1963-1964). New York, NY: Atlas Publishing Company.

Sterling, D. A., Lewis, R. D., Luke, D. A., \& Shadel, B. N. (2000). A portable X-ray fluorescence instrument for analyzing dust wipe samples for lead: Evaluation with field samples doi:https://doi.org/10.1006/enrs.2000.4058

Sullivan M. (2015). More evidence of unpublished industry studies of lead smelter/refinery workers. International journal of occupational and environmental health, 21(4), 308-313. doi:10.1179/2049396715Y.0000000010

Thermo Elemental handbook of elemental analysis (2001). AAS, GFAAS, ICP or ICP-MS? Which technique should I use? An elementary overview of elemental analysis. Available at: http://oliver.chemistry.ucsc.edu/122/Lab5\%20Handout.pdf

ThermoFisher Scientific (2015), Technology focus: X-ray Fluorescence (XRF) in Mining. https://www.thermofisher.com/blog/mining/technology-focus-X-ray-fluorescence-xrf-inmining/. (Accessed Dec 7, 2019).

Troesken W, Beeson PE. (2003). The significance of lead water mains in American cities. Some historical evidence. In Health and Labor Force Participation over the Life Cycle: Evidence from the Past, ed. DL Costa, pp. 181-202. Chicago: Univ. Chicago Press

Troesken W. (2006). The Great Lead Water Pipe Disaster. Cambridge, MA: MIT Press

US Department of Housing and Urban Development (1995). Guidelines for the evaluation and control of lead-based paint hazards in housing. Washington, D.C.

U.S Environmental Protection Agency (EPA). (2017). America's Children and the Environment, $3^{\text {rd }}$ Edition. https://www.epa.gov/ace/ace-biomonitoring-lead

U.S. Environmental Protection Agency (EPA). (2018). Lead at Superfund Sites. https://www.epa. gov/superfund/lead-superfund-sites

U.S. Environmental Protection Agency (EPA). (2018). Flint Drinking Water Response. https://www.epa.gov/flint

Vorvolakos, T., Arseniou, S., \& Samakouri, M. (2016). There is no safe threshold for lead exposure: A literature review. Psychiatrike = Psychiatriki, 27(3), 204-214. Retrieved from http://0search.ebscohost.com.lib.utep.edu/login.aspx?direct=true $\& \mathrm{db}=\mathrm{cmedm} \& \mathrm{AN}=27837574 \&$ $\underline{\text { site }=\text { ehost-live } \& \text { scope }=\text { site }}$

Van Loon, J. C. (1985), Selected Methods of Trace Metal Analysis (John Wiley and Sons, New York). 
Welz, B., and Sterling, M. (1999) Atomic absorption spectrometry. 3rd ed. Wiley-VCH, Weinheim, Germany

Welz B, Becker-Ross H, Florek S, Heitmann U, Vale MGR. (2003) High-resolution continuumsource atomic absorption spectrometry - What can we expect? J Braz Chem Soc.;14:220-229.

Welz, B., H. Becker-Ross, S. Florek, and U. Heitmann. (2005). High-resolution continuum source AAS: The better way to do atomic absorption spectrometry. Darmstadt: GmbH, Wiley-Vch.

Welz, B., M. G. R. Vale, D. L. G. Borges, and U. Heitmann. (2007) Progress in direct solid sampling analysis using line source and high-resolution continuum source electrothermal atomic absorption spectrometry. Anal. Bio anal. Chem. 389: 2085 - 2095.

Weitzman M., Baten A., Rosenthal D. G., Hoshino R., Tohn E., Jacobs D. E. (2013). Housing and child health. Curr. Probl. Pediatr. Adolesc. Health Care 43:187-224., doi:https://doi.org/10.1016/j.cppeds.2013.06.001

Zahran S, Laidlaw MAS, McElmurry SP, Filippelli GM, Taylor M. (2013). Linking source and effect: resuspended soil lead, air lead, and children's blood lead levels in Detroit, Michigan. Environ. Sci. Technol.47:2839-45

https://www.epa.gov/newsreleases/epa-administrator-pruitt-invites-nations-leaders-join-epaefforts-reduce-childhood-lead

https://www.epa.gov/lead/protect-your-family-exposures-lead

https://www.cdc.gov/nceh/lead/tips.htm 


\section{APPENDIX}

Appendix A. Table of results from XRF and ICPMS measurements of dust wipe samples for lead

\begin{tabular}{|c|c|c|c|}
\hline SAMPLE ID & LOCATION & XRF $\left.\left(\mu g / f^{2}\right)^{2}\right)$ & ICPMS $\left(\mu \mathrm{g} / \mathrm{ft}^{2}\right)$ \\
\hline 75-058-1-02 & Floor & 1725.88 & 2256.40 \\
\hline 75-058-1-01 & Windowsill & 74.04 & 78.63 \\
\hline $75-058-1-03$ & Floor & 74.55 & 137.63 \\
\hline 75-058-1-04 & Windowsill & 918.76 & 1863.20 \\
\hline $75-058-1-06$ & Floor & 252.41 & 435.18 \\
\hline 75-058-1-07 & Windowsill & 1337.39 & 1826.10 \\
\hline $75-058-1-08$ & Floor & 15.75 & 18.16 \\
\hline 75-058-1-09 & Floor & 5367.85 & 7173.90 \\
\hline $75-058-1-10$ & Windowsill & 4982.06 & 6005.90 \\
\hline 75-058-1-11 & Floor & 6083.35 & 6432.10 \\
\hline 75-047-1-01 & Floor & 0 & 1.39 \\
\hline 75-047-1-03 & Floor & 0 & 0.81 \\
\hline 75-047-1-04 & Floor & 0 & 0.95 \\
\hline 75-047-1-05 & Floor & 0 & 0.50 \\
\hline 75-047-1-06 & Floor & 0 & 1.27 \\
\hline 75-047-1-07 & Floor & 0 & 0.13 \\
\hline 75-047-1-09 & Floor & 0 & 0.37 \\
\hline $75-047-1-10$ & Floor & 5.32 & 0.03 \\
\hline $75-047-1-11$ & Floor & 5.25 & 0.48 \\
\hline 75-048-1-01 & Floor & 0 & 0.31 \\
\hline $75-048-1-02$ & Floor & 0 & 0.85 \\
\hline 75-048-1-04 & Windowsill & 13.90 & 20.98 \\
\hline $75-048-1-05$ & Windowsill & 4.11 & 9.88 \\
\hline $75-048-1-06$ & Windowsill & 0 & 1.65 \\
\hline 75-048-1-07 & Windowsill & 0 & 1.37 \\
\hline 75-048-1-08 & Floor & 0 & 0.28 \\
\hline 75-048-1-09 & Floor & 0 & 0.51 \\
\hline $75-048-1-10$ & Windowsill & 10.93 & 12.03 \\
\hline 75-038-1-01 & Floor & 0 & 0.22 \\
\hline $75-038-1-02$ & Windowsill & 50.28 & 89.43 \\
\hline 75-038-1-03 & Windowsill & 626.70 & 1717.5 \\
\hline 75-019-1-01 & Floor & 3.17 & 7.43 \\
\hline $75-019-1-02$ & Floor & 0 & 3.42 \\
\hline 75-019-1-03 & Floor & 0.85 & 4.89 \\
\hline 75-019-1-05 & Windowsill & 38.88 & 101.18 \\
\hline 75-019-1-06 & Floor & 0.25 & 5.12 \\
\hline
\end{tabular}




\begin{tabular}{|c|c|c|c|}
\hline $75-019-1-07$ & Floor & 0 & 1.69 \\
\hline $75-016-1-01$ & Floor & 0 & 0.45 \\
\hline $75-016-1-02$ & Floor & 0 & 0.75 \\
\hline $75-076-1-02$ & Floor & 5.57 & 4.89 \\
\hline $75-076-1-03$ & Floor & 5.34 & 1.53 \\
\hline $75-076-1-04$ & Floor & 6.04 & 8.33 \\
\hline $75-076-1-05$ & Windowsill & 5.52 & 3.70 \\
\hline $75-076-1-06$ & Floor & 5.06 & 2.95 \\
\hline $75-076-1-07$ & Floor & 5.66 & 3.02 \\
\hline $75-076-1-08$ & Floor & 4.90 & 1.37 \\
\hline $75-076-1-09$ & Floor & 4.99 & 1.13 \\
\hline $75-076-1-10$ & Floor & 14.32 & 21.04 \\
\hline $75-076-1-11$ & Floor & 5.49 & 6.21 \\
\hline $75-076-1-12$ & Windowsill & 5.40 & 2.47 \\
\hline $75-076-1-13$ & Floor & 5.15 & 2.38 \\
\hline $75-076-1-16$ & Windowsill & 5.62 & 5.50 \\
\hline $75-003-1-02$ & Floor & 5.27 & 0.42 \\
\hline $75-003-1-03$ & Floor & 5.19 & 1.50 \\
\hline $75-003-1-04$ & Floor & 5.27 & 1.22 \\
\hline $75-003-1-05$ & Windowsill & 23.21 & 135.90 \\
\hline $75-003-1-06$ & Floor & 5.01 & 0.56 \\
\hline $75-003-1-08$ & Floor & 5.11 & 0.74 \\
\hline $75-003-1-09$ & Windowsill & 6.46 & 4.84 \\
\hline $75-003-1-10$ & Floor & 5.41 & 3.87 \\
\hline $75-058-1-01 b$ & Windowsill & 4.99 & 0.67 \\
\hline $75-058-1-02 b$ & Floor & 9.81 & 16.68 \\
\hline $75-058-1-03 b$ & Floor & 6.33 & 11.66 \\
\hline $75-058-1-04 b$ & Windowsill & 5.05 & 1.20 \\
\hline $75-058-1-06 b$ & Floor & 5.70 & 0.87 \\
\hline $75-058-1-07 b$ & Windowsill & 5.11 & 1.78 \\
\hline $75-058-1-08 b$ & Floor & 7.29 & 10.71 \\
\hline $75-058-1-09 b$ & Floor & 5.84 & 5.37 \\
\hline $75-058-1-10 b$ & Windowsill & 10.17 & 11.34 \\
\hline $75-058-1-11 b$ & Floor & 6.63 & 9.87 \\
\hline $75-058-1-12$ & Floor & 22.35 & 22.93 \\
\hline $75-058-1-14$ & Windowsill & 10.03 & 9.70 \\
\hline $75-034-1-01$ & Floor & 5.09 & 2.45 \\
\hline $75-034-1-02$ & Floor & 5.27 & 2.01 \\
\hline $75-034-1-03$ & Windowsill & 5.35 & \\
\hline $75-034-1-04$ & & & 46.25 \\
\hline $75-034-1-05$ & & & \\
\hline
\end{tabular}




\begin{tabular}{|c|c|c|c|}
\hline $75-034-1-07$ & Floor & 4.96 & 0.64 \\
\hline $75-029-1-01$ & Floor & 4.90 & 0.04 \\
\hline $75-029-1-02$ & Floor & 4.97 & 0.19 \\
\hline $75-029-1-03$ & Windowsill & 6.19 & 10.78 \\
\hline $75-029-1-04$ & Windowsill & 5.58 & 13.71 \\
\hline $75-029-1-05$ & Floor & 5.11 & 0.52 \\
\hline $75-029-1-06$ & Floor & 5.07 & 0.39 \\
\hline $75-029-1-07$ & Floor & 5.35 & 1.49 \\
\hline $75-097-1-01$ & Floor & 5.13 & 0.24 \\
\hline $75-097-1-02$ & Floor & 4.99 & 0.24 \\
\hline $75-097-1-03$ & Floor & 5.08 & 0.19 \\
\hline $75-097-1-04$ & Windowsill & 5.70 & 2.24 \\
\hline $75-097-1-05$ & Floor & 4.91 & 0.36 \\
\hline $75-097-1-06$ & Windowsill & 5.36 & 3.10 \\
\hline $75-097-1-07$ & Floor & 4.83 & 0.25 \\
\hline $75-038-1-01 b$ & Floor & 5.13 & 0.50 \\
\hline $75-038-1-02 b$ & Windowsill & 5.78 & 7.37 \\
\hline $75-038-1-03 b$ & Windowsill & 59.95 & 67.75 \\
\hline $75-106-01-01$ & Floor & 5.19 & 0.72 \\
\hline $75-106-01-02$ & Windowsill & 103.80 & 260.5 \\
\hline $75-106-01-03$ & Floor & 5.36 & 1.76 \\
\hline $75-106-01-04$ & Windowsill & 66.36 & 134.3 \\
\hline $75-106-01-05$ & Floor & 5.30 & 1.31 \\
\hline $75-106-01-06$ & Windowsill & 100.8 & 233.40 \\
\hline $75-106-01-07$ & Floor & 48.59 & 69.31 \\
\hline $75-106-01-08$ & Floor & 39.67 & 73.46 \\
\hline $75-091-01-01$ & Floor & 5.19 & 0.29 \\
\hline $75-091-01-02$ & Floor & 4.87 & 0.21 \\
\hline $75-091-01-03$ & Floor & 4.84 & 0.83 \\
\hline $75-091-01-04$ & Floor & 5.03 & 0.18 \\
\hline
\end{tabular}


Appendix B. Description of dust wipe collection

1) Wear booties before sampling home.

2) Record on the Home Environment Sampling Log Sheet the Date, Home ID, Home Address, Contact information of home participant, names of UTEP staff during home testing, and type of samples.

3) Identify sampling area. Sampling area can be walls, floors, windowsills, or window troughs.

4) Wear gloves before measuring the sampling area of interest.

5) Outline the sampling area with the 12 by 12 -inch template and secure with tape

6) Do not touch the sampling area. For windowsills and window troughs, measure areas that will give you a sample area of 12 by 12 inches. If not possible then the sampling area is the area of the window trough or windowsill.

7) Measure both dimensions of the frame

8) Record sample ID, location, surface type, and dimensions (length and width to calculate area) of the sample area in inches on the Home Environment Sampling Log Sheet. For windowsills and window troughs, measure and record the area (length and width to calculate area).

9) Pre-label a plastic bag with the corresponding sample ID, date, and staff initials. Make sure to label on one side and top of the plastic bag.

10) Tear open a lead dust sampling wipe and dispose of wrapper.

11) Be sure the wipe is moist. If the wipe is dry do not use. Do not touch the wipe with bare hands. Unfold completely the wipe with gloved hands. 
12) First wipe the identified sampling area side-to-side in an S-like motion, pressing firmly with the palm.

\section{Always attempt to include all visible dust, and do not cross boundary tape.}

13) Fold the wipe in half with the contaminated side facing inward.

\section{Always make sure not to spill dust when folding.}

14) Make the second wipe pass from top to bottom also in an S-like motion.

15) Fold the wipe again in half with the contaminated side facing inward.

16) Make the third wipe pass around the perimeter of the sampled area.

17) Fold the wipe again in half with the contaminated side facing inward.

18) Insert the folded dust sample wipe into the corresponding labelled plastic bag.

19) Remove gloves and place into the trash bag.

20) Proceed to sampling other areas if needed. Always change gloves between samples.

Clean up after sampling:

1) After completing dust sampling (all samples needed were collected), remove gloves, shoe covers, and tape and place into the trash bag.

2) Check your clothing and shoes (especially soles) before leaving the home. If you see dust on clothes or sole of shoes, wipe with a clean baby wipe. Place dirty baby wipe in trash bag.

3) Wash your face and hands with warm soapy water. 


\section{CURRICULUM VITA}

Alexander Boakye Obeng was born in Kumasi, a city in Ghana, West Africa. He is a second generation college student and attended the University of Cape Coast (UCC) in Ghana. At UCC he conducted research on assessing the level of knowledge and perception of a community on childhood lead poisoning which received high evaluations from notable members from the metropolitan health directorate and the metropolitan assembly. He graduated in 2013 with a Bachelor of Science degree in Environmental Science. After graduation, he worked as the Environmental health officer at Ahmadiyya Muslim Mission Hospital for 3 years. During this time, he educated the hospital community regarding environmental health risks and maintaining a safe environment. In 2017, Alex was accepted into the Master of Public Health program at the University of Texas at El Paso (UTEP). From the start of his master's program, Alex was part of an interdisciplinary research team working on a neighborhood level strategy to eliminate lead exposures in El Paso, Texas with funding from the department of Housing and Urban Development (HUD). Alex oversaw the group's home and environmental investigations using a portable X-Ray Fluorescence (pXRF) device. He was also responsible for training the team members on the safe use of the device both on and off the field and took the lead in creating an educational brochure for educating participants on lead exposure and prevention. Alex plans to pursue a doctoral degree in environmental and occupational health. Afterwards, he plans to work with research institutions in Ghana that are committed to promoting and eradicating the plethora of environmental health issues in the country. He aspires to teach and continue to conduct research at a university in Ghana where he hopes to impart knowledge onto the next generation of environmental and occupational health professionals in Ghana.

Email address: alexoboakye@gmail.com 